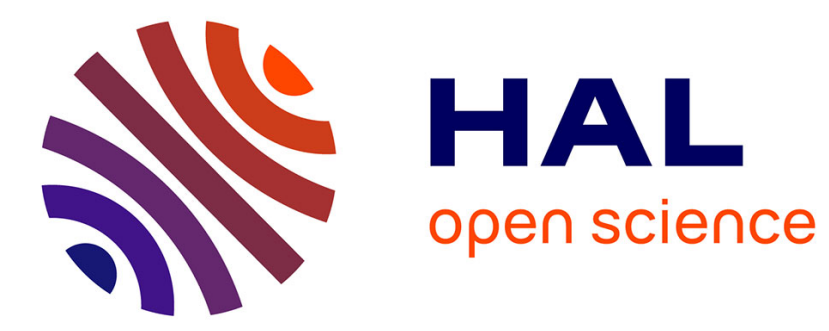

\title{
Null controllability of Kolmogorov-type equations
}

Karine Beauchard

\section{To cite this version:}

Karine Beauchard. Null controllability of Kolmogorov-type equations. Mathematics of Control, Signals, and Systems, 2014, 26 (1), pp.145-176. 10.1007/s00498-013-0110-x . hal-00826117

\section{HAL Id: hal-00826117 https://hal.science/hal-00826117}

Submitted on 26 May 2013

HAL is a multi-disciplinary open access archive for the deposit and dissemination of scientific research documents, whether they are published or not. The documents may come from teaching and research institutions in France or abroad, or from public or private research centers.
L'archive ouverte pluridisciplinaire HAL, est destinée au dépôt et à la diffusion de documents scientifiques de niveau recherche, publiés ou non, émanant des établissements d'enseignement et de recherche français ou étrangers, des laboratoires publics ou privés. 


\title{
Null controllability of Kolmogorov-type equations
}

\author{
K. BEAUCHARD ${ }^{* \dagger}$
}

\begin{abstract}
We study the null controllability of Kolmogorov-type equations $\partial_{t} f+$ $v^{\gamma} \partial_{x} f-\partial_{v}^{2} f=u(t, x, v) 1_{\omega}(x, v)$ in a rectangle $\Omega$, under an additive control supported in an open subset $\omega$ of $\Omega$.

For $\gamma=1$, with periodic-type boundary conditions, we prove that null controllability holds in any positive time, with any control support $\omega$. This improves the previous result [5], in which the control support was a horizontal strip.

With Dirichlet boundary conditions and a horizontal strip as control support, we prove that null controllability holds in any positive time if $\gamma=1$, or if $\gamma=2$ and $\omega$ contains the segment $\{v=0\}$, and only in large time if $\gamma=2$ and $\omega$ does not contain the segment $\{v=0\}$.

Our approach, inspired from [7,31], is based on 2 key ingredients: the observability of the Fourier components of the solution of the adjoint system, uniformly with respect to the frequency, and the explicit exponential decay rate of these Fourier components.
\end{abstract}

Key words: null controllability, degenerate parabolic equations, Carleman estimates, hypoelliptic systems.

\section{Introduction}

\subsection{Main result}

We consider Kolmogorov-type equations

$$
\partial_{t} f+v^{\gamma} \partial_{x} f-\partial_{v}^{2} f=u(t, x, v) 1_{\omega}(x, v), \quad(t, x, v) \in(0,+\infty) \times \Omega,
$$

where $\gamma \in \mathbb{N}^{*}, \Omega=\mathbb{T} \times(-1,1), \mathbb{T}$ is the $1 \mathrm{D}$-torus, $\omega$ is an open subset of $\Omega, 1_{\omega}$ is the characteristic function of this set and $u(t, x, v)$ is a source term located on the subdomain $\omega$. It is a linear control system in which the state is $f$ and the control $u$ is supported in the subset $\omega$.

Depending on the value of $\gamma$, we use different boundary conditions in variable $v$ : periodic type boundary conditions when $\gamma=1$

$$
\begin{cases}f(t, x-t,-1)=f(t, x+t,+1), & (t, x) \in(0,+\infty) \times \mathbb{T}, \\ \partial_{v} f(t, x-t,-1)=\partial_{v} f(t, x+t, 1), & (t, x) \in(0,+\infty) \times \mathbb{T},\end{cases}
$$

*CMLS, Ecole Polytechnique, 91128 Palaiseau Cedex, France, email: Karine.Beauchard@math.polytechnique.fr

†The author was partially supported by the "Agence Nationale de la Recherche" (ANR), Projet Blanc EMAQS number ANR-2011-BS01-017-01. 
or Dirichlet boundary conditions when $\gamma \in \mathbb{N}^{*}$

$$
f(t, x,-1)=f(t, x,+1)=0, \quad(t, x) \in(0,+\infty) \times \mathbb{T} .
$$

We will also use initial data

$$
f(0, x, v)=f_{0}(x, v), \quad(x, v) \in \Omega .
$$

Definition 1 (Null controllability). Let $T>0$ and $\gamma \in \mathbb{N}^{*}$. System (1)-(2) (resp. System (1)-(3)) is null controllable in time $T$ if, for every $f_{0} \in L^{2}(\Omega)$, there exists $u \in L^{2}((0, T) \times \Omega)$ such that the solution of the Cauchy problem (1)-(2)-(4) (resp. (1)-(3)-(4)) satisfies $f(T, \cdot, \cdot)=0$.

When $\gamma=1$ and $\omega=\mathbb{T} \times(a, b)$ for some $a, b \in(-1,1)$, the null controllability of system (1)-(2) is proved in [5]. The goals of this article are

1. to improve the strategy of [5] in order to conclude with more general control supports, in the case of periodic-type boundary conditions when $\gamma=1$ (i.e. for system (1)-(2)),

2. to study the case of Dirichlet boundary conditions (i.e. system (1)-(3)),

3. to give an indication about the possible critical parameter $\gamma$ for the null controllability (possibly $\gamma=2$ ), for system (1)-(3), as for Grushin equations in [4].

The main results of this paper are the following ones.

Theorem 1. 1. If $\gamma=1$ and $\omega$ is an open subset of $\Omega$, then the system (1)-(2) is null controllable in any time $T>0$.

2. If $\gamma=1$ and $\omega=\mathbb{T} \times(a, b)$ with $-1<a<b<1$, then the system (1)-(3) is null controllable in any time $T>0$.

3. If $\gamma=2$ and $\omega=\mathbb{T} \times(a, b)$ with $0<a<b<1$ then there exists $T^{*} \geqslant a^{2} / 2$ such that

- the system (1)-(3) is null controllable in any time $T>T^{*}$,

- the system (1)-(3) is not null controllable in time $T<T^{*}$.

4. If $\gamma=2$ and $\omega=\mathbb{T} \times(a, b)$ with $-1<a<0<b<1$ then the system (1)-(3) is null controllable in any time $T>0$.

Note that in the third statement, the set $\{v=0\}$ is not contained in the control location $\omega$, contrary to the fourth case. Theorem 1 emphasizes several behaviors:

1. a sensitivity to boundary conditions (see the asymptotic behavior of Fourier components in Propositions 2, 10 and 17),

2. a finite speed of propagation through the set $\{v=0\}$ with $\gamma=2$ and Dirichlet boundary conditions. 
By duality, Theorem 1 is equivalent to observability results for the adjoint system

$$
\partial_{t} g-v^{\gamma} \partial_{x} g-\partial_{v}^{2} g=0, \quad(t, x, v) \in(0,+\infty) \times \Omega,
$$

associated to the following boundary conditions when $\gamma=1$

$$
\begin{cases}g(t, x-T+t,-1)=g(t, x+T-t, 1), & (t, x) \in(0,+\infty) \times \mathbb{T}, \\ \partial_{v} g(t, x-T+t,-1)=\partial_{v} g(t, x+T-t, 1), & (t, x) \in(0,+\infty) \times \mathbb{T},\end{cases}
$$

or the following ones for $\gamma \in \mathbb{N}^{*}$

$$
g(t, x,-1)=g(t, x, 1)=0, \quad(t, x) \in(0,+\infty) \times \mathbb{T} .
$$

We will also use initial data

$$
g(0, x, v)=g_{0}(x, v), \quad(x, v) \in \Omega .
$$

Definition 2 (Observability). Let $T>0$ and $\gamma \in \mathbb{N}^{*}$. System (5)-(6) (resp. System (5)-(7)) is observable in $\omega$ in time $T$ if there exists $C>0$ such that, for every $g_{0} \in L^{2}(\Omega)$, the solution of the Cauchy problem (5)-(6)-(8) (resp. (5)-(7)-(8)) satisfies

$$
\int_{\Omega}|g(T, x, v)|^{2} d x d v \leqslant C \int_{0}^{T} \int_{\omega}|g(t, x, v)|^{2} d x d v d t .
$$

Theorem 2. 1. If $\gamma=1$ and $\omega$ is an open subset of $\Omega$, then the system (5)-(6) is observable in $\omega$ in any time $T>0$.

2. If $\gamma=1$ and $\omega=\mathbb{T} \times(a, b)$ with $0<a<b<1$, then the system (5)-(7) is observable in $\omega$ in any time $T>0$.

3. If $\gamma=2$ and $\omega=\mathbb{T} \times(a, b)$ with $0<a<b<1$, then there exists $T^{*} \geqslant a^{2} / 2$ such that

- the system (5)-(7) is observable in $\omega$ in any time $T>T^{*}$,

- the system (5)-(7) is not observable in $\omega$ in time $T<T^{*}$.

4. If $\gamma=2$ and $\omega=\mathbb{T} \times(a, b)$ with $-1<a<0<b<1$ then the system (5)-(7) is observable in $\omega$ in any time $T>0$.

Remark 1. Let us emphasize that, when $\gamma=2, \omega=\mathbb{T} \times(a, b)$ with $0<a<$ $b<1$ and $T \leqslant T^{*}$, then unique continuation holds for system (5)-(7), i.e. any solution $g$ of (5)-(7) satisfies

$$
g \equiv 0 \text { on }(0, T) \times \omega \Rightarrow g \equiv 0 \text { on }(0, T) \times \Omega
$$

(see Proposition 9 for a proof).

\subsection{Motivation and bibliographical comments}

\subsubsection{Null controllability of the heat equation}

The null and approximate controllability of the heat equation are essentially well understood subjects for both linear and semilinear equations, for bounded or unbounded domains (see, for instance, [16], [19], [21], [22], [23], [26], [30], 
[31], [33], [36], [37], [42], [43]) and also with discontinuous (see, e.g. [17], [6], [7], [39]) or singular ([40] and [18]) coefficients.

In particular, the heat equation on a smooth bounded domain $\Omega$ of $\mathbb{R}^{d}(d \in$ $\mathbb{N}^{*}$ ), with a source term located on an open subset $\omega$ of $\Omega$ is null controllable in arbitrarily small time $T$ and with an arbitrarily small control support $\omega$. This result is due, for the case $d=1$, to H. Fattorini and D. Russell [20, Theorem 3.3], and, for $d \geqslant 2$, to O. Imanuvilov [28], [29] (see also the book [25] by A. Fursikov and O.Imanuvilov) and G. Lebeau and L. Robbiano [31]. It is then natural to wonder whether the same result holds for degenerate parabolic equations.

\subsubsection{Boundary-degenerate parabolic equations}

The null controllability of parabolic equations degenerating on the boundary of the domain in one space dimension is well-understood, much less so in higher dimension. Given $0<a<b<1$ and $\gamma>0$, let us consider the 1D equation

$$
\partial_{t} w+\partial_{x}\left(x^{2 \gamma} \partial_{x} w\right)=u(t, x) 1_{(a, b)}(x), \quad(t, x) \in(0, \infty) \times(0,1),
$$

with suitable boundary conditions. Then, null controllability holds if and only if $\gamma \in(0,1)$ (see $[13,14]$ ), while, for $\gamma \geq 1$, the best result one can show is "regional null controllability"(see [12]), which consists in controlling the solution within the domain of influence of the control. Several extensions of the above results are available in one space dimension, see $[1,34]$ for equations in divergence form, $[11,10]$ for nondivergence form operators, and [9, 24] for cascade systems. Fewer results are available for multidimensional problems, mainly in the case of two dimensional parabolic operators which simply degenerate in the normal direction to the boundary of the space domain, see [15].

\subsubsection{Parabolic equations degenerating inside the domain}

In [35], the authors study linearized Crocco type equations

$$
\begin{cases}\partial_{t} f+\partial_{x} f-\partial_{v v} f=u(t, x, v) 1_{\omega}(x, v), & (t, x, v) \in(0, T) \times \mathbb{T} \times(0,1), \\ f(t, x, 0)=f(t, x, 1)=0, & (t, x) \in(0, T) \times \mathbb{T} .\end{cases}
$$

For a given strict open subset $\omega$ of $\mathbb{T} \times(0,1)$, they prove that null controllability does not hold: the optimal result is regional null controllability. Note that, for Kolmogorov equation (1), the coupling between the diffusion (in $v$ ) and the transport (in $x$ at speed $v$ ) generates diffusion both in variables $x$ and $v$ (see Propositions 2, 10 and 17).

In [4], we study Grushin-type equations

$$
\begin{cases}\partial_{t} f-\partial_{x}^{2} f-|x|^{2 \gamma} \partial_{y}^{2} f=u(t, x, y) 1_{\omega}(x, y), & (t, x, y) \in(0, T) \times \Omega, \\ f(t, x, y)=0, & (t, x, y) \in(0, T) \times \partial \Omega,\end{cases}
$$

where $\Omega:=(-1,1) \times(0,1), \omega \subset(0,1) \times(0,1)$, and $\gamma>0$. Here, the parabolic operator degenerates along the line $\{0\} \times(0,1)$. We prove that

- null controllability holds in any time $T>0$ when $\gamma \in(0,1)$,

- null controllability does not hold (whatever $T>0$ ) when $\gamma>1$, 
- when $\gamma=1$ and $\omega=(a, b) \times(0,1)$ with $0<a<b<1$, there exists $T_{\min } \geqslant a^{2} / 2$ such that null controllability holds when $T>T_{\min }$ and does not hold when $T<T_{\min }$.

Note that, contrary to Grushin-type equations (9), in Kolmogorov equations (1), the parabolic operator degenerates everywhere on the domain.

\subsubsection{Null controllability and hypoellipticity}

It could be interesting to analyze the connections between null controllability and hypoellipticity.

We recall that a linear differential operator $P$ with $C^{\infty}$ coefficients in an open set $\Omega \subset \mathbb{R}^{d}$ is called hypoelliptic if, for every distribution $u$ in $\Omega, u$ must be a $C^{\infty}$ function in every open set where so is $P u$. The following sufficient condition (which is also essentially necessary) for hypoellipticity is due to Hörmander (see $[27])$.

Theorem 3. Let $P$ be a second order differential operator of the form $P=$ $\sum_{j=1}^{r} X_{j}^{2}+X_{0}+c$, where $X_{0}, \ldots, X_{r}$ denote first order homogeneous differential operators in an open set $\Omega \subset \mathbb{R}^{n}$ with $C^{\infty}$ coefficients, and $c \in C^{\infty}(\Omega)$. Assume that there exists $n$ operators among

$$
X_{j_{1}},\left[X_{j_{1}}, X_{j_{2}}\right],\left[X_{j_{1}},\left[X_{j_{2}}, X_{j_{3}}\right]\right], \ldots,\left[X_{j_{1}},\left[X_{j_{2}},\left[X_{j_{3}},\left[\ldots, X_{j_{k}}\right] \ldots\right]\right]\right],
$$

where $j_{i} \in\{0,1, \ldots, r\}$, which are linearly independent at any given point in $\Omega$. Then, $P$ is hypoelliptic.

The Kolmogorov operator $K:=v^{\gamma} \partial_{x}+\partial_{v}^{2}$ satisfies Hörmander condition for every $\gamma \in \mathbb{N}^{*}$. Indeed, $K=X_{0}+X_{1}^{2}$ where

$$
X_{0}(x, v):=\left(\begin{array}{c}
v^{\gamma} \\
0
\end{array}\right), \quad X_{1}(x, v):=\left(\begin{array}{l}
0 \\
1
\end{array}\right)
$$

and

$$
\left[X_{0}, X_{1}\right](x, v)=\left(\begin{array}{c}
\gamma v^{\gamma-1} \\
0
\end{array}\right), \quad\left[X_{1},\left[X_{1}, X_{2}\right]\right](x, v)=\left(\begin{array}{c}
\gamma(\gamma-1) v^{\gamma-2} \\
0
\end{array}\right) .
$$

Thus, when $\gamma=1$, the first iterated Lie bracket is sufficient, whereas when $\gamma=2$, the second one the required (at $v=0$ ), to satisfy Hörmander's condition.

First, we emphasize that hypoellipticity is not sufficient for unique continuation. For instance, Alinhac and Zuily built a zero order $C^{\infty}$-perturbation of the Kolmogorov operator $K$ for which unique continuation does not hold: there exists $C^{\infty}$-functions $u(t, x, v)$ and $a(t, x, v)$ on a neighborhood $V$ of 0 in $\mathbb{R}^{3}$ such that $K u+a u=0, u(t, x, v)=v(t, x, v)=0$ when $v<0$, and $0 \in \operatorname{Supp}(u)$ [2]. Therefore, hypoellipticity cannot be sufficient neither for null controllability.

Let us recall that the Grushin operator $G:=\partial_{x}^{2}+|x|^{2 \gamma} \partial_{y}^{2}$ is the hypoelliptic operator of type II associated to the vector field $\left(X_{0}, X_{1}\right)$ (i.e. $\left.G=X_{0}^{2}+X_{1}^{2}\right)$, whereas Kolmogorov operator $K$ is the one of type I (i.e. $K=X_{0}+X_{1}^{2}$ ). Both are prototypes of hypoelliptic operators. 
For Grushin-type equations, null controllability (with control on a vertical strip) holds only when the first iterated Lie-bracket is sufficient to satisfy Hörmander's condition $(\gamma \in(0,1])$. For Kolmogorov-type equations, null controllability (with control on a horizontal strip) holds when the two first iterated Lie-brackets are sufficient $(\gamma \in\{1,2\})$. A general result which relates null controllability of hypoelliptic operators (depending on their type) to the number of iterated Lie brackets that are necessary to satisfy Hörmander's condition would be very interesting, but remains - for the time being - a challenging open problem.

This article also underlines an important influence of the boundary conditions on the validity of null controllability, through the exponential decay rate of Fourier components (see Propositions 2 and 17).

\subsection{Structure of the article}

In Section 2, we state a global Carleman estimate, for 1D heat equations with parameters, which is a preliminary result for the whole article. In Section 3, we prove Theorem 1 for $\gamma=1$ with periodic type boundary conditions. In Section 4 , we study the well posedness and the Fourier decomposition of the solutions of (1)-(3) when $\gamma \in\{1,2\}$. In Section 5 (resp. 6), we prove Theorem 1 for $\gamma=2$ (resp. $\gamma=1$ ), with Dirichlet boundary conditions.

\section{Preliminary}

The goal of this section is the statement of a global Carleman estimate for the Fourier components (in $x$ ) of the solution of the adjoint system (5). For $n \in \mathbb{Z}$ and $\gamma \in \mathbb{N}^{*}$, we introduce the operator

$$
\mathcal{P}_{n, \gamma} g:=\partial_{t} g+i n v^{\gamma} g-\partial_{v}^{2} g .
$$

Proposition 1. We assume $\gamma \in \mathbb{N}^{*}$ (resp. $\gamma=1$ ). Let $a, b$ be such that $-1<a<b<1$. There exist a weight function $\beta \in C^{1}\left([-1,1], \mathbb{R}_{+}^{*}\right)$, positive constants $\mathcal{C}_{1}, \mathcal{C}_{2}$ such that, for every $n \in \mathbb{Z}, \gamma \in\{1,2\}, T>0$ and $g \in C^{0}\left([0, T], L^{2}(-1,1)\right) \cap L^{2}\left(0, T ; H_{0}^{1}(-1,1)\right)$ (resp. $g \in C^{0}\left([0, T], L^{2}(-1,1)\right) \cap$ $L^{2}\left(0, T ; H^{1}(-1,1)\right)$ such that $g(t,-1)=g(t, 1) e^{i 2 n(T-t)}$ and $\left.\partial_{v} g(t,-1)=\partial_{v} g(t,+1) e^{i 2 n(T-t)}\right)$ the following inequality holds

$$
\begin{aligned}
& \mathcal{C}_{1} \int_{0}^{T} \int_{-1}^{1}\left(\frac{M}{t(T-t)}\left|\frac{\partial g}{\partial v}(t, v)\right|^{2}+\frac{M^{3}}{(t(T-t))^{3}}|g(t, v)|^{2}\right) e^{-\frac{M \beta(v)}{t(T-t)}} d v d t \\
& \leqslant \int_{0}^{T} \int_{-1}^{1}\left|\mathcal{P}_{n, \gamma} g\right|^{2} e^{-\frac{M \beta(v)}{t(T-t)}} d v d t+\int_{0}^{T} \int_{a}^{b} \frac{M^{3}}{(t(T-t))^{3}}|g(t, v)|^{2} e^{-\frac{M \beta(v)}{t(T-t)}} d v d t
\end{aligned}
$$

where $M:=\mathcal{C}_{2} \max \left\{T+T^{2} ; \sqrt{|n|} T^{2}\right\}$.

The proof of this estimate is classical (see [25]): our weight $\beta$ is the usual one (see (36), (37), (38) and (39)). We only track carefully the behavior with respect to $n$ of the different constants. For sake of completeness, a proof is reproduced in Appendix, in the case of Dirichlet boundary conditions on $g$. For periodic-type boundary conditions, one may use a periodic weight function $\beta$, as in [5]. 


\section{Proof of Theorem 1 with $\gamma=1$ and periodic- type boundary conditions}

In all this section, we take $\gamma=1$.

\subsection{Well posedness, Fourier decomposition and dissipa- tion}

We have the following well posedness result, for the Cauchy-problem (1)-(2).

Proposition 2. Let $T>0, f_{0} \in L^{2}(\Omega)$ and $u \in L^{2}((0, T) \times \Omega)$. There exists a unique solution $f \in C^{0}\left([0, T], L^{2}(\Omega)\right)$ of the Cauchy problem (1)-(2)-(4). Moreover, if $u \equiv 0$, the Fourier components

$$
f_{n}(t, v):=\int_{\mathbb{T}} f(t, x, v) e^{-i n x} d x, \quad t \in(0,+\infty), v \in(-1,1), n \in \mathbb{Z}
$$

satisfy

$$
\left\|f_{n}(t, .)\right\|_{L^{2}(-1,1)} \leqslant\left\|f_{n}(0, .)\right\|_{L^{2}(-1,1)} e^{-\frac{n^{2} t^{3}}{12}}, \forall t>0, n \in \mathbb{Z} .
$$

Proof of Proposition 2: The function $h(t, x, v):=f(t, x+v t, v)$ solves a linear equation with coefficients depending only on $t$, and periodic boundary conditions. Thus, we have an explicit expression

$$
\begin{aligned}
& h(t, x, v)=\sum_{p, n \in \mathbb{Z}} \hat{f}(n, p) e^{-(p \pi)^{2} t+n p \pi t^{2}-n^{2} \frac{t^{3}}{3}} e^{i(n x+p \pi v)} \\
& +\sum_{p, n \in \mathbb{Z}}\left(\int_{0}^{t} \hat{w}(\tau, n, p) e^{(p \pi)^{2} \tau-n p \pi \tau^{2}+n^{2} \frac{\tau^{3}}{3}}\right) e^{-(p \pi)^{2} t+n p \pi t^{2}-n^{2} \frac{t^{3}}{3}} e^{i(n x+p \pi v)}
\end{aligned}
$$

where

$$
\begin{gathered}
\hat{f}(n, p):=\int_{\mathbb{T}} \int_{-1}^{1} f_{0}(x, v) e^{-i(n x+p \pi v)} d v d x, \\
\hat{w}(\tau, n, p):=\int_{\mathbb{T}} \int_{-1}^{1} 1_{\omega}(x+v t, v) u(\tau, x+v t, v) e^{-i(n x+p \pi v)} d v d x .
\end{gathered}
$$

The dissipation result is a consequence of the relation

$$
-(p \pi)^{2} t+n p \pi t^{2}-n^{2} \frac{t^{3}}{3}=-t\left(p \pi-\frac{n t}{2}\right)^{2}-\frac{n^{2} t^{3}}{12} .
$$

We refer to [5] for more details.

\subsection{Null controllability of initial data with a finite number of Fourier modes}

The goal of this section is the proof of the following result.

Proposition 3. There exists $C>0$ such that, for every $T>0, N \in \mathbb{N}^{*}$ and $f_{0} \in L^{2}(\Omega)$ of the form $f_{0}(x, v)=\sum_{|n| \leqslant N} f_{0, n}(v) e^{i n x}$ there exists a control $u \in L^{2}((0, T) \times \Omega)$ such that the solution of (1)-(2)-(4) satisfies $f(T, .,)=$. and

$$
\|u\|_{L^{2}((0, T) \times \Omega)} \leqslant T e^{C\left(1+\frac{1}{T}+N\right)}\left\|f_{0}\right\|_{L^{2}(\Omega)} .
$$


By duality, this null controllability result is equivalent to the following observability inequality.

Proposition 4. There exists $C>0$ such that, for every $T>0, N \in \mathbb{N}^{*}$ and $g_{0} \in L^{2}(\Omega)$ of the form $g_{0}(x, v)=\sum_{|n| \leqslant N} g_{0, n}(v) e^{i n x}$ the solution of (5)-(6)-(8) satisfies

$$
\int_{\Omega}|g(T, x, v)|^{2} d x d v \leqslant T^{2} e^{C\left(1+\frac{1}{T}+N\right)} \int_{0}^{T} \int_{\omega}|g(t, x, v)|^{2} d x d v .
$$

For the proof of Proposition 4, we need the 2 following ingredients. The first one is a classical inequality, proved, for example, in [31] (see also [32]).

Proposition 5. Let $c, d \in \mathbb{R}$ be such that $c<d$. There exists $C>0$ such that, for every $N \in \mathbb{N}^{*}$ and $\left(b_{n}\right)_{|n| \leqslant N} \in \mathbb{R}^{2 N+1}$,

$$
\sum_{n=-N}^{N}\left|b_{n}\right|^{2} \leqslant e^{C N} \int_{c}^{d}\left|\sum_{n=-N}^{N} b_{n} e^{i n x}\right|^{2} d x .
$$

The second ingredient is an estimate of the observability constant for the Fourier components of $g$.

Proposition 6. Let $a, b \in \mathbb{R}$ be such that $-1<a<b<1$. There exists $C>0$ such that, for every $T>0, n \in \mathbb{Z}, g_{0, n} \in L^{2}(-1,1)$, the solution of

$$
\begin{cases}\partial_{t} g_{n}+i n v g_{n}-\partial_{v}^{2} g_{n}=0, & (t, v) \in(0,+\infty) \times(-1,1), \\ g_{n}(t,-1)=g_{n}(t,+1) e^{i 2 n(T-t)}, & t \in(0,+\infty), \\ \partial_{v} g_{n}(t,-1)=\partial_{v} g_{n}(t,+1) e^{i 2 n(T-t)}, & t \in(0,+\infty), \\ g_{n}(0, v)=g_{0, n}(v), & v \in(-1,1),\end{cases}
$$

satisfies

$$
\int_{-1}^{1}\left|g_{n}(T, v)\right|^{2} d v \leqslant T^{2} e^{C\left(1+\frac{1}{T}+\sqrt{|n|}\right)} \int_{0}^{T} \int_{a}^{b}\left|g_{n}(t, v)\right|^{2} d v d t .
$$

Proof of Proposition 6: For $t \in(T / 3,2 T / 3)$, we have

$$
\frac{4}{T^{2}} \leqslant \frac{1}{t(T-t)} \leqslant \frac{9}{2 T^{2}}
$$

and

$$
\int_{-1}^{1}\left|g_{n}(T, v)\right|^{2} d v \leqslant \int_{-1}^{1}\left|g_{n}(t, v)\right|^{2} d v .
$$

Thanks to Proposition 1, we get

$$
\mathcal{C}_{1} \frac{64 M^{3}}{T^{6}} e^{-\frac{9 M \beta^{*}}{2 T^{2}}} \frac{T}{3} \int_{0}^{1}\left|g_{n}(T, v)\right|^{2} d v \leqslant \mathcal{C}_{3} \int_{0}^{T} \int_{a}^{b}\left|g_{n}(t, v)\right|^{2} d v d t
$$

where $\beta^{*}:=\max \{\beta(x) ; x \in[-1,1]\}, \beta_{*}:=\min \{\beta(x) ; x \in[-1,1]\}$ and $\mathcal{C}_{3}:=$ $\max \left\{x^{3} e^{-\beta_{*} x} ; x \in[-1,1]\right\}$. Using the inequality $M \geqslant \mathcal{C}_{2}\left[T+T^{2}\right]$, we get

$$
\int_{0}^{1}\left|g_{n}(T, v)\right|^{2} d v \leqslant \mathcal{C}_{4} T^{2} e^{c_{1} \frac{M}{T^{2}}} \int_{0}^{T} \int_{a}^{b}\left|g_{n}(t, v)\right|^{2} d v d t
$$


for some constants $c_{1}, \mathcal{C}_{4}>0$ (independent of $n, T$ and $g_{0, n}$ ).

First case: $\sqrt{|n|}<1+\frac{1}{T}$. Then, $M=\mathcal{C}_{2}\left(T+T^{2}\right)$ thus

$$
\int_{-1}^{1}\left|g_{n}(T, v)\right|^{2} d v \leqslant \mathcal{C}_{4} T^{2} e^{c_{1} \mathcal{C}_{2}\left(1+\frac{1}{T}\right)} \int_{0}^{T} \int_{a}^{b}\left|g_{n}(t, v)\right|^{2} d v d t
$$

Second case: $\sqrt{|n|} \geqslant 1+\frac{1}{T}$. Then, $M=\mathcal{C}_{2} \sqrt{|n|} T^{2}$, thus

$$
\int_{0}^{1}\left|g_{n}(T, v)\right|^{2} d v \leqslant \mathcal{C}_{4} T^{2} e^{c_{1} \mathcal{C}_{2} \sqrt{|n|}} \int_{0}^{T} \int_{a}^{b}\left|g_{n}(t, v)\right|^{2} d v d t
$$

This gives the conclusion.

Now, let us prove Proposition 4, thanks to Propositions 5 and 6 .

Proof of Proposition 4: Let $a, b, c, d \in \mathbb{R}$ be such that $a<b, c<d$ and $(c, d) \times(a, b) \subset \omega$. Let $g_{n}$ be the solution of (11) for $n=-N, \ldots, N$. Then $g(t, x, v)=\sum_{|n| \leqslant N} g_{n}(t, v) e_{n}(x)$, where $e_{n}(x):=e^{i n x}$. From the orthogonality of the family $\left(e_{n}\right)_{n \in \mathbb{Z}}$ in $L^{2}(\mathbb{T})$, Propositions 6 and 5 , we deduce

$$
\begin{aligned}
\int_{\Omega} g(T, x, v)^{2} d x d v & =\sum_{|n| \leqslant N} \int_{-1}^{1}\left|g_{n}(T, v)\right|^{2} d v \\
& \leqslant T^{2} e^{C\left(1+\frac{1}{T}+\sqrt{N}\right)} \sum_{|n| \leqslant N} \int_{0}^{T} \int_{a}^{b}\left|g_{n}(t, v)\right|^{2} d v d t \\
& \leqslant T^{2} e^{C\left(N+\frac{1}{T}+\sqrt{N}\right)} \int_{0}^{T} \int_{a}^{b} \int_{c}^{d}\left|\sum_{|n| \leqslant N} g_{n}(t, v) e_{n}(x)\right|^{2} d x d v d t \\
& \leqslant T^{2} e^{C\left(N+\frac{1}{T}\right)} \int_{0}^{T} \int_{\omega}|g(t, x, v)|^{2} d x d v d t
\end{aligned}
$$

where the constant $C$ may change from line to line.

\subsection{Construction of the control function}

The goal of this section is the proof of the statement 1 of Theorem 1. The construction of the control is the one of [7] (itself inspired from [31], see also $[32])$.

For $n \in \mathbb{Z}$, we define $e_{n}(x):=e^{i n x}$ and $H_{n}:=e_{n} \otimes L^{2}(0,1)$, which is a closed subspace of $L^{2}(\Omega)$. For $j \in \mathbb{N}$, we define $E_{j}:=\oplus_{|n| \leqslant 2^{j}} H_{n}$ and $\Pi_{E_{j}}$ the orthogonal projection from $L^{2}(\Omega)$ to $E_{j}$.

Let $T>0$ and $f_{0} \in L^{2}(\Omega)$ and let us build a control $u \in L^{2}((0, T) \times \Omega)$ such that the solution of (1)-(3)-(4) satisfies $f(T)=0$. Let $\rho \in \mathbb{R}$ with

$$
0<\rho<\frac{1}{3}
$$

Let $K=K(\rho)>0$ be such that $K \sum_{j=1}^{\infty} 2^{-j \rho}=T$. Let $\left(a_{j}\right)_{j \in \mathbb{N}}$ be defined by $a_{0}=0, a_{j+1}=a_{j}+2 T_{j}$ where $T_{j}:=K 2^{-j \rho}$ for every $j \in \mathbb{N}$. We now define the 
control function $u$ in the following way. On $\left[a_{j}, a_{j}+T_{j}\right]$, we apply a control $u$ such that $\Pi_{E_{j}} f\left(a_{j}+T_{j}\right)=0$ and

$$
\|u\|_{L^{2}\left(\left(a_{j}, a_{j}+T_{j}\right) \times \Omega\right)} \leqslant \mathcal{C}_{j}\left\|f\left(a_{j}\right)\right\|_{L^{2}(\Omega)}
$$

where $\mathcal{C}_{j}:=T_{j} e^{C\left(2^{j}+\frac{1}{T_{j}}\right)}$ (see Proposition 3). Then

$$
\left\|f\left(a_{j}+T_{j}\right)\right\|_{L^{2}(\Omega)} \leqslant\left(1+\sqrt{T_{j}} \mathcal{C}_{j}\right)\left\|f\left(a_{j}\right)\right\|_{L^{2}(\Omega)} .
$$

On $\left[a_{j}+T_{j}, a_{j+1}\right]$, we apply no control, to take advantage of the dissipation of the solution proved in Proposition 2

$$
\left\|f\left(a_{j+1}\right)\right\|_{L^{2}(\Omega)} \leqslant e^{-2^{2 j} \frac{T_{j}^{3}}{12}}\left\|f\left(a_{j}+T_{j}\right)\right\|_{L^{2}(\Omega)} .
$$

Thus, we obtain

$$
\left\|f\left(a_{j+1}\right)\right\|_{L^{2}(\Omega)} \leqslant e^{\sum_{k=1}^{2^{j}} \ln \left(1+\sqrt{T_{k}} \mathcal{C}_{k}\right)-2^{2 k} \frac{T_{k}^{3}}{12}}\left\|f_{0}\right\|_{L^{2}(\Omega)} .
$$

The choice of $\rho$ ensures that the sum in the exponent tends to $-\infty$ when $j \rightarrow$ $+\infty$, this gives $f(T)=0$. Arguing in the same way, one proves that the control built above belongs to $L^{2}((0, T) \times \Omega)$.

\section{With Dirichlet boundary conditions: well posed- ness, Fourier decomposition, unique continua- tion}

In this section $\gamma \in\{1,2\}$. Let

$$
\mathcal{V}:=\left\{f \in C^{\infty}(\mathbb{T} \times(-1,1)) ; \exists K \subset(-1,1) \text { compact s.t. } \operatorname{Supp}(f) \subset \mathbb{T} \times K\right\} .
$$

For $f \in \mathcal{V}$, we define

$$
|f|_{V}:=\left(\int_{\Omega}\left|\partial_{v} f(x, v)\right|^{2} d x d v\right)^{1 / 2}
$$

and $V:=\operatorname{Adh}_{||_{V}}(\mathcal{V})$. Observe that $H_{0}^{1}(\Omega) \subset V \subset L^{2}(\Omega)$, thus $V$ is dense in $L^{2}(\Omega)$. We define the operator $A_{\gamma}$ by

$$
\begin{aligned}
D\left(A_{\gamma}\right):= & \left\{f \in V ;-\partial_{v}^{2} f+v^{\gamma} \partial_{x} f \in L^{2}(\Omega)\right\}, \\
& A_{\gamma} f:=-\partial_{v}^{2} f+v^{\gamma} \partial_{x} f .
\end{aligned}
$$

Then $D\left(A_{\gamma}\right)$ is dense in $L^{2}(\Omega),\left(A_{\gamma}, D\left(A_{\gamma}\right)\right)$ is closed and both $A_{\gamma}$ and $A_{\gamma}^{*}$ are dissipative, thus $\left(A_{\gamma}, D\left(A_{\gamma}\right)\right)$ generates an strongly continuous semigroup $S_{\gamma}(t)$ of contractions of $L^{2}(\Omega)$ (see Lumer-Phillips theorem [38, Corollary 4.4, Chapter 1, page 15], or HilleYosida theorem [8, Theorem VII.4, page 105]). For every $T>0, u \in L^{2}((0, T) \times \Omega), f_{0} \in L^{2}(\Omega)$, the weak solution of (1)-(3)-(4) is

$$
f(t)=S_{\gamma}(t) f_{0}+\int_{0}^{t} S_{\gamma}(t-s)\left(1_{\omega} u\right)(s) d s
$$

and the following existence and uniqueness result follows. 
Proposition 7. Let $\gamma \in\{1,2\}$. For every $T>0, u \in L^{2}((0, T) \times \Omega), f_{0} \in L^{2}(\Omega)$ there exists a unique weak solution $f \in C^{0}\left([0, T], L^{2}(\Omega)\right) \cap L^{2}((0, T), V)$ of (1)(3)-(4). Moreover, $f(t) \in D\left(A_{\gamma}\right)$ and $\partial_{t} f(t) \in L^{2}(\Omega)$ for a.e. $t \in(0, T)$.

Let us consider a solution of (5)-(7)-(8) in the sense above. Since $g \in$ $C^{0}\left([0, T], L^{2}(\Omega)\right)$, the function $x \mapsto g(t, x, v)$ belongs to $L^{2}(\mathbb{T})$ for almost every $(t, v) \in[0,+\infty) \times(-1,1)$, thus, it can be developed in Fourier series of $x$ as follows

$g(t, x, v)=\sum_{n \in \mathbb{Z}} g_{n}(t, v) e^{i n x} \quad$ where $\quad g_{n}(t, v):=\int_{\mathbb{T}} g(t, x, v) e^{-i n x} d x, \quad \forall n \in \mathbb{Z}$.

Proposition 8. For every $n \in \mathbb{Z}, g_{n}$ is the unique solution of

$$
\begin{cases}\partial_{t} g_{n}-i n v^{\gamma} g_{n}-\partial_{v}^{2} g_{n}=0, & (t, v) \in(0,+\infty) \times(-1,1) \\ g_{n}(t, \pm 1)=0, & t \in(0,+\infty) \\ g_{n}(0, v)=g_{0, n}(v), & v \in(-1,1)\end{cases}
$$

where $g_{0, n} \in L^{2}(-1,1)$ is given by

$$
g_{0, n}(v):=\int_{\mathbb{T}} g_{0}(x, v) e^{-i n x} d x, \quad v \in(-1,1)
$$

This result may be proved by following the same steps as in [4, Section 2.2]. Then, the following unique continuation property follows.

Proposition 9. Let $\gamma \in\{1,2\}, \omega=\mathbb{T} \times(a, b)$ where $0<a<b<1, T>0$ and $g \in C^{0}\left([0, T], L^{2}(\Omega)\right) \cap L^{2}((0, T), V)$ a solution of $(5)-(7)$. If $g \equiv 0$ on $(0, T) \times \omega$, then $g \equiv 0$ on $(0, T) \times \Omega$.

Proof of Proposition 9: Let $n \in \mathbb{Z}$ and $g_{n}$ be defined by (14). Then $g_{n} \equiv 0$ on $(0, T) \times(a, b)$ and $g_{n}$ solves $(15)$. Thus, Proposition 1 ensures that $g_{n} \equiv 0$ on $(0, T) \times(-1,1)$. Therefore, $g \equiv 0$ on $(0, T) \times \Omega$.

\section{Proof of Theorem 1 when $\gamma=2$}

In all this section, we take $\gamma=2$ and $\omega=\mathbb{T} \times(a, b)$ where $-1<a<b<1$. In the first 4 subsections, we prove the statement 3 of Theorem 1 and in the last subsection, we prove the statement 4 .

\subsection{Dissipation speed on $(-1,1)$}

The goal of this section is the proof of the following dissipation property.

Proposition 10. There exists $K, \delta>0$ such that, for every $n \in \mathbb{Z}-\{0\}$ and $g_{0, n} \in H^{1}(-1,1)$, the solution of (15) satisfies

$$
\int_{-1}^{1}\left|g_{n}(t, v)\right|^{2} d v \leqslant K e^{-\delta \sqrt{|n|} t} \int_{-1}^{1}\left(\frac{1}{\sqrt{n}}\left|\partial_{v} g_{0, n}(v)\right|^{2}+\sqrt{n}\left|v g_{0, n}(v)\right|^{2}\right) d v, \forall t>0 .
$$

The proof of Proposition 10 relies on the following result. 
Proposition 11. There exists $A, B, C, \delta>0$ with $B^{2}<A C$ such that, for every $L>0$ and $h_{0} \in H^{1}(-L, L)$, the solution of

$$
\begin{cases}\partial_{\tau} h=\partial_{y}^{2} h+i y^{2} h, & (\tau, y) \in(0,+\infty) \times(-L, L) \\ h(\tau, \pm L)=0, & \tau \in(0,+\infty) \\ h(0, y)=h_{0}(y), & y \in(-L, L)\end{cases}
$$

satisfies

$$
\mathcal{L}(t) \leqslant \mathcal{L}(0) e^{-\delta \tau}, \forall \tau>0
$$

where

$\mathcal{L}(\tau)=\int_{-L}^{L}\left(|h(\tau, y)|^{2}+A\left|\partial_{y} h(\tau, y)\right|^{2}-2 B \Im\left[y \overline{h(\tau, y)} \partial_{y} h(\tau, y)\right]+C|y h(\tau, y)|^{2}\right) d y$.

Proof of Proposition 11: This proof is inspired from [41]. Let $A, B, C>0$ be such that

$$
B^{2}<A C \quad \text { and } \quad A^{2}+C^{2}<\frac{B}{2}
$$

(for instance $A=\epsilon \tilde{A}, B=\epsilon \tilde{B}, C=\epsilon \tilde{C}$ for any $\tilde{A}, \tilde{B}, \tilde{C}, \epsilon>0$ such that $\tilde{B}^{2}<\tilde{A} \tilde{C}$ and $\left.\epsilon\left(\tilde{A}^{2}+\tilde{C}^{2}\right)<\tilde{B} / 2\right)$. Easy computations give

$$
\begin{aligned}
\frac{1}{2} \frac{d \mathcal{L}}{d \tau}= & -3 B\|y h\|^{2}-\left\|\partial_{y} h\right\|^{2}-C\left\|y \partial_{y} h\right\|^{2}-A\left\|\partial_{y}^{2} h\right\|^{2} \\
& +C\|h\|^{2}-2 A \Im\left[\int_{-L}^{L} y \partial_{y} \bar{h} h\right]-2 B \Im\left[\int_{-L}^{L} y \partial_{y}^{2} \bar{h} \partial_{y} h\right] .
\end{aligned}
$$

where $\|\cdot\|$ is the usual $L^{2}((-L, L), \mathbb{C})$-norm, i.e.

$$
\|f\|:=\int_{-L}^{L}|f(y)|^{2} d y .
$$

Thanks to the following inequalities

$$
\begin{gathered}
C\|h\|^{2} \leqslant 2 C\|y h\|\left\|\partial_{y} h\right\| \leqslant \frac{B}{2}\|y h\|^{2}+\frac{2 C^{2}}{B}\left\|\partial_{y} h\right\|^{2}, \\
-2 A \Im\left[\int_{0}^{L} y \partial_{y} \bar{h} h\right] \leqslant \frac{B}{2}\|y h\|^{2}+\frac{2 A^{2}}{B}\left\|\partial_{y} h\right\|^{2}, \\
-2 B \Im\left[\int_{0}^{L} y \partial_{y}^{2} \bar{h} \partial_{y} h\right] \leqslant A\left\|\partial_{y}^{2} h\right\|^{2}+\frac{B^{2}}{A}\left\|y \partial_{y} h\right\|^{2},
\end{gathered}
$$

we get

$$
\frac{1}{2} \frac{d \mathcal{L}}{d \tau} \leqslant-2 B\|y h\|^{2}-\left(1-\frac{2\left(A^{2}+C^{2}\right)}{B}\right)\left\|\partial_{y} h\right\|^{2} .
$$

Thanks to (18), there exists $\delta>0$ (independent of $L$ ) such that $\frac{d \mathcal{L}}{d \tau} \leqslant-\delta \mathcal{L}$, which gives the conclusion.

Proof of Proposition 10: One may assume that $n>0$, otherwise, consider $\overline{g_{n}}$. In order to simplify the notations, we write $g$, instead of $g_{n}$. The function $h(\tau, y)$ defined by

$$
g(t, v)=h(\sqrt{n} t, \sqrt[4]{n} v)
$$

satisfies (16) with $L=\sqrt[4]{n}$ and $h_{0}(y):=g_{0, n}(y / \sqrt[4]{n})$. From the previous proposition, we know that

$$
\widetilde{\mathcal{L}}(t)=\int_{-1}^{1}\left(|g(t, v)|^{2}+\frac{A}{\sqrt{n}}\left|\partial_{v} g(t, v)\right|^{2}-2 B \Im\left[v \overline{g(t, v)} \partial_{v} g(t, v)\right]+C \sqrt{n}|v g(t, v)|^{2}\right) d v
$$


satisfies $\widetilde{\mathcal{L}}(t) \leqslant \widetilde{\mathcal{L}}(0) e^{-\delta \sqrt{n} t}$. Moreover, using (18) and

$$
\|g\|^{2} \leqslant 2\|v g\|\left\|\partial_{v} g\right\| \leqslant \sqrt{n}\|v g\|^{2}+\frac{1}{\sqrt{n}}\left\|\partial_{v} g\right\|^{2}
$$

we get

$$
\widetilde{\mathcal{L}}(0) \leqslant \int_{-1}^{1}\left(\frac{2 A+1}{\sqrt{n}}\left|\partial_{v} g_{0}(v)\right|^{2}+(2 C+1) \sqrt{n}\left|v g_{0}(v)\right|^{2}\right) d v
$$

Thus

$$
\begin{aligned}
\int_{-1}^{1}\left|g_{n}(t, v)\right|^{2} d v & \leqslant \widetilde{\mathcal{L}}(t) \\
& \leqslant \widetilde{\mathcal{L}}(0) e^{-\delta \sqrt{n} t} \\
& \leqslant K \int_{-1}^{1}\left(\frac{1}{\sqrt{n}}\left|\partial_{v} g_{0}(v)\right|^{2}+\sqrt{n}\left|v g_{0}(v)\right|^{2}\right) d v e^{-\delta \sqrt{n} t}
\end{aligned}
$$

where $K:=\max \{2 A+1 ; 2 C+1\}$.

\subsection{Null controllability in large time $T$ when $0<a<b<1$}

In this section, we assume $0<a<b<1$. Our goal is to prove the existence of a time $T_{1}>0$ such that, for every $T>T_{1}$, the system (5)-(7) is observable in $\omega$ in time $T$. The following uniform observability result gives the conclusion.

Proposition 12. There exists $T_{1}, C>0$ such that for every $T>T_{1}, n \in \mathbb{Z}$ and $g_{0, n} \in L^{2}(-1,1)$, the solution of (15) satisfies

$$
\int_{-1}^{1} g_{n}(T, v)^{2} d v \leqslant C \int_{0}^{T} \int_{a}^{b} g_{n}(t, v)^{2} d v d t .
$$

Proof of Proposition 12: Working as in the proof of Proposition 6, we get

$$
\mathcal{C}_{3} e^{-c^{*} \sqrt{n}} \int_{T / 3}^{2 T / 3} \int_{-1}^{1}\left(\sqrt{n}\left|\partial_{v} g\right|^{2}+n^{3 / 2}|g|^{2}\right) d v d t \leqslant \mathcal{C}_{4} \int_{0}^{T} \int_{a}^{b}|g|^{2} d v d t
$$

for $n$ large enough, where $\mathcal{C}_{3}:=\mathcal{C}_{2} \max \left\{4 \mathcal{C}_{1} ;\left(4 \mathcal{C}_{1}\right)^{3}\right\}, c^{*}:=\frac{9}{2} \mathcal{C}_{2} \max \{\beta(v) ; v \in$ $[-1,1]\}, \mathcal{C}_{4}:=\max \left\{x^{3} e^{-\beta_{*} x} ; x \geqslant 0\right\}$ and $\beta_{*}:=\min \{\beta(v) ; v \in(a, b)\}$. Moreover, thanks to Proposition 10, we have, for any $t \in(T / 3,2 T / 3)$,

\section{REPRENDRE ICI}

$$
\begin{aligned}
& \int_{-1}^{1}\left(\sqrt{n}\left|\partial_{v} g(t, v)\right|^{2}+n^{3 / 2}|g(t, v)|^{2}\right) d v \\
\geqslant & n \int_{-1}^{1}\left(\frac{1}{\sqrt{n}}\left|\partial_{v} g(t, v)\right|^{2}+\sqrt{n}|v g(t, v)|^{2}\right) d v \\
\geqslant & \frac{n}{K} e^{\delta \sqrt{n} \frac{T}{3}} \int_{-1}^{1}|g(T, v)|^{2} d v .
\end{aligned}
$$

Thus,

$$
\int_{-1}^{1}|g(T)|^{2} d v \leqslant \frac{1}{n T} \mathcal{C}_{5} e^{\left[c^{*}-\delta T / 3\right] \sqrt{n}} \int_{0}^{T} \int_{a}^{b}|g|^{2} d v d t
$$

where $\mathcal{C}_{5}:=3 K \mathcal{C}_{4} / \mathcal{C}_{3}$. This gives the conclusion with $T_{1}:=3 c^{*} / \delta$. 


\subsection{No null controllability when $0<a<b<1$ and $T \leqslant a^{2} / 2$}

In this section, we assume $0<a<b<1$. The goal of this section is to prove that (5)-(7) is not observable in $\omega$ in time $T \leqslant a^{2} / 2$, which is equivalent to the following non uniform observability result.

Proposition 13. Let $T \leqslant a^{2} / 2$. For every $C>0$, there exists $n \in \mathbb{Z}, g_{0, n} \in$ $L^{2}(-1,1)$ such that the solution of $(15)$ satisfies

$$
\int_{-1}^{1} g_{n}(T, v)^{2} d v>C \int_{0}^{T} \int_{a}^{b} g_{n}(t, v)^{2} d v d t .
$$

\section{Proof of Proposition 13:}

First step: Approximate solution. Let $\epsilon>0$ be such that $b<1-\epsilon$ and $\theta \in C^{\infty}(\mathbb{R})$ be such that

$$
\operatorname{Supp}(\theta) \subset(-1-\epsilon,-1+\epsilon) \cup(1-\epsilon, 1+\epsilon), \quad \theta( \pm 1)=1 .
$$

For $n \in \mathbb{N}^{*}$, we define

$$
\tilde{g}_{n}(t, v):=\sqrt[8]{n}\left(e^{-\sqrt{i n} \frac{v^{2}}{2}}-e^{-\frac{\sqrt{i n}}{2}} \theta(v)\right) e^{-\sqrt{i n} t}
$$

where $\sqrt{i}:=e^{i \frac{\pi}{4}}$. We have

$$
\left\{\begin{array}{l}
\partial_{t} \tilde{g}_{n}+i n v^{2} \tilde{g}_{n}-\partial_{v}^{2} \tilde{g}_{n}=\sqrt[8]{n} e^{-\frac{\sqrt{i n}}{2}}\left(\sqrt{i n} \theta+\theta^{\prime \prime}-i n v^{2} \theta\right) e^{-\sqrt{i n} t}, \\
\tilde{g}_{n}(t, \pm 1)=0 .
\end{array}\right.
$$

Let $g_{n}$ be the solution of

$$
\begin{cases}\partial_{t} g_{n}+i n v^{2} g_{n}-\partial_{v}^{2} g_{n}=0, & (t, v) \in(0, T) \times(0,1), \\ g_{n}(t, \pm 1)=0, & t \in(0, T), \\ g_{n}(0, v)=\tilde{g}_{n}(0, v), & v \in(0,1) .\end{cases}
$$

We have

$$
\begin{aligned}
& \frac{1}{2} \frac{d}{d t}\left\|\left(\tilde{g}_{n}-g_{n}\right)(t)\right\|_{L^{2}(-1,1)}^{2}=-\left\|\partial_{v}\left(\tilde{g}_{n}-g_{n}\right)(t)\right\|_{L^{2}(-1,1)}^{2} \\
& +\Re\left(\int_{-1}^{1} \sqrt[8]{n} e^{-\frac{\sqrt{i n}}{2}}\left(\sqrt{i n} \theta(v)+\theta^{\prime \prime}(v)-i n v^{2} \theta(v)\right) e^{-\sqrt{i n} t} \frac{\left.\tilde{g}_{n}-g_{n}\right)(t, v)}{(v)} .\right.
\end{aligned}
$$

Thanks to Poincare and Cauchy-Schwarz inequalities, we get

$$
\frac{d}{d t}\left\|\left(\tilde{g}_{n}-g_{n}\right)(t)\right\|_{L^{2}(-1,1)} \leqslant-C_{P}\left\|\left(\tilde{g}_{n}-g_{n}\right)(t)\right\|_{L^{2}(-1,1)}+C_{1} n^{9 / 8} e^{-\frac{\sqrt{2 n}}{4}} e^{-\frac{\sqrt{2 n} t}{2}}
$$

where $C_{P}$ is the Poincare constant on $(-1,1)$ and $C_{1}$ is a positive constant that depends only on $\theta$. Thus

$$
\left\|\left(\tilde{g}_{n}-g_{n}\right)(t)\right\|_{L^{2}(-1,1)} \leqslant C_{2} n^{5 / 8} e^{-\frac{\sqrt{2 n}}{4}}, \forall t \geqslant 0 .
$$

where $C_{2}>0$ does not depend on $n$.

Second step: Conclusion. Let $T \leqslant a^{2} / 2$. Working by contradiction, we assume that there exists $C_{T}>0$ such that, for every $n \in \mathbb{N}^{*}$,

$$
\int_{-1}^{1} g_{n}(T, v)^{2} d v \leqslant C_{T} \int_{0}^{T} \int_{a}^{b} g_{n}(t, v)^{2} d v d t
$$


Thanks to the triangular inequality and (20), we deduce that

$$
\begin{aligned}
\left\|\tilde{g}_{n}(T)\right\|_{L^{2}(-1,1)} \leqslant & \left(C_{T} \int_{0}^{T} \int_{a}^{b}\left|\tilde{g}_{n}\right|^{2} d v d t\right)^{1 / 2}+\left\|\left(\tilde{g}_{n}-g_{n}\right)(T)\right\|_{L^{2}(-1,1)} \\
& +\left(C_{T} \int_{0}^{T} \int_{a}^{b}\left|\tilde{g}_{n}-g_{n}\right|^{2} d v d t\right)^{1 / 2} \\
\leqslant & \left(C_{T} \int_{0}^{T} \int_{a}^{b}\left|\tilde{g}_{n}\right|^{2} d v d t\right)^{1 / 2}+\left(1+\sqrt{T C_{T}}\right) C_{2} n^{5 / 8} e^{-\frac{\sqrt{2 n}}{4}} .
\end{aligned}
$$

However, there exists $C_{3}, C_{4}>0$ such that, when $n \rightarrow+\infty$,

$$
\begin{gathered}
\left\|\tilde{g}_{n}(T)\right\|_{L^{2}} \sim C_{3} e^{-\frac{\sqrt{2 n} T}{2}} \\
\left(\int_{0}^{T} \int_{a}^{b}\left|\tilde{g}_{n}(t, v)\right|^{2} d v d t\right)^{1 / 2} \sim \frac{C_{4}}{n^{3 / 8}} e^{-\frac{\sqrt{2 n} a^{2}}{4}},
\end{gathered}
$$

which gives a contradiction.

\subsection{End of the proof of Theorems 1.3 and 2.3}

Let us consider $\gamma=2$ and $\omega=\mathbb{T} \times(a, b)$ with $0<a<b<1$. From Proposition 12 and Bessel Parseval equality, we know that system (5)-(7) is observable in $\omega$ in any time $T>T_{1}$. From Proposition 13, we deduce that for any time $T \leqslant \frac{a^{2}}{2}$, (5)-(7) is not observable in $\omega$ in time $T$. Thus, the quantity

$$
T^{*}:=\inf \{T>0 ; \text { system }(5)(7) \text { is observable in } \omega \text { in time } T\}
$$

is well defined and belongs to $\left[\frac{a^{2}}{2},+\infty\right)$. Clearly, observability in some time $T_{\sharp}$ implies observability in any time $T>T_{\sharp}$, so

- for every $T>T^{*},(5)-(7)$ is observable in $\omega$ in time $T$,

- for every $T<T^{*},(5)-(7)$ is not observable in $\omega$ in time $T$.

\subsection{Null controllability in any time $T>0$ when $a<0<b$}

In this section, $\omega=\mathbb{T} \times(-a, a)$ where $a>0$. We fix $\beta \in(0, a)$. Our goal is the proof of the statement 4 of Theorem 1, thanks to a cut-off argument.

\subsubsection{Preliminary}

We define $\Omega_{1}:=\mathbb{T} \times(\beta, 1), \omega_{1}:=\mathbb{T} \times(\beta, a)$ and we consider the system

$$
\begin{cases}\partial_{t} f+v^{2} \partial_{x} f-\partial_{v}^{2} f=u(t, x, v) 1_{\omega_{1}}(x, v), & (t, x, v) \in(0, T) \times \Omega_{1}, \\ f(t, x, \beta)=f(t, x, 1)=0, & (t, x) \in(0, T) \times \mathbb{T},\end{cases}
$$

The goal of this section is the proof of the following result.

Proposition 14. The system (21) is null controllable in any time $T>0$.

As in section 5.2, this is equivalent to the following observability result. 
Proposition 15. There exists $C>0$ such that, for every $n \in \mathbb{Z}$ and $g_{0, n} \in$ $L^{2}(\beta, 1)$, the solution of

$$
\begin{cases}\partial_{t} g_{n}-i n v^{2} g_{n}-\partial_{v}^{2} g_{n}=0, & (t, v) \in(0,+\infty) \times(\beta, 1), \\ g_{n}(t, \beta)=g_{n}(t, 1)=0, & t \in(0,+\infty), \\ g_{n}(0, v)=g_{0, n}(v), & v \in(\beta, 1),\end{cases}
$$

satisfies

$$
\int_{\beta}^{1}\left|g_{n}(T, v)\right|^{2} d v \leqslant C \int_{0}^{T} \int_{\beta}^{a}\left|g_{n}(t, v)\right|^{2} d v d t
$$

For the proof of Proposition 15, we need the following dissipation result.

Proposition 16. There exists $K, \delta>0$ such that, for every $n \in \mathbb{Z}, g_{0, n} \in$ $H^{1}(\beta, 1)$, the solution of (22) satisfies

$$
\int_{\beta}^{1}\left|g_{n}(t, v)\right|^{2} d v \leqslant K e^{-\delta|n|^{2 / 3} t} \int_{\beta}^{1}\left(\left|g_{0, n}(v)\right|^{2}+\frac{1}{|n|^{2 / 3}}\left|\partial_{v} g_{0, n}(v)\right|^{2}\right) d v .
$$

Proof of Proposition 16: One may assume that $n>0$, otherwise, consider $\overline{g_{n}}$. To simplify the notations, we write $g$, instead of $g_{n}$. Let $B, C>0$ be such that

$$
B^{2}<2 C \text { and } 3 B>4 C^{2}
$$

(for instance $B=\sqrt{C}$ with $C>0$ small enough). The function

$$
\mathcal{L}(t):=\int_{\beta}^{1}\left(|g(t, v)|^{2}+\frac{B}{n^{1 / 3}} \Im\left[v g(t, v) \overline{\partial_{v} g(t, v)}\right]+\frac{C}{n^{2 / 3}}\left|\partial_{v} g(t, v)\right|^{2}\right) d v
$$

satisfies

$$
\begin{aligned}
\frac{1}{2} \frac{d \mathcal{L}}{d t}= & -\frac{3 B n^{2 / 3}}{2}\|v g\|^{2}-\left\|\partial_{v} g\right\|^{2}-\frac{C}{n^{2 / 3}}\left\|\partial_{v}^{2} g\right\|^{2} \\
& -2 C n^{1 / 3} \Im\left[\int_{\beta}^{1} v g \partial_{v} \bar{g}\right]-\frac{B}{n^{1 / 3}} \Im\left[\int_{\beta}^{1} v \partial_{v} g \partial_{v}^{2} \bar{g}\right] .
\end{aligned}
$$

Thanks to

$$
\begin{gathered}
-2 C n^{1 / 3} \Im\left[\int_{\beta}^{1} v g \partial_{v} \bar{g}\right] \leqslant \frac{1}{2}\left\|\partial_{v} g\right\|^{2}+2 C^{2} n^{2 / 3}\|v g\|^{2}, \\
-\frac{B}{n^{1 / 3}} \Im\left[\int_{\beta}^{1} v \partial_{v} g \partial_{v}^{2} \bar{g}\right] \leqslant \frac{C}{n^{2 / 3}}\left\|\partial_{v}^{2} g\right\|^{2}+\frac{B^{2}}{4 C}\left\|\partial_{v} g\right\|^{2},
\end{gathered}
$$

we get

$$
\frac{1}{2} \frac{d \mathcal{L}}{d t} \leqslant-\left(\frac{3 B}{2}-2 C^{2}\right) n^{2 / 3} \beta^{2}\|g\|^{2}-\left(\frac{1}{2}-\frac{B^{2}}{4 C}\right)\left\|\partial_{v} g\right\|^{2} .
$$

Thanks to (23), there exists $\delta>0$ (independent of $n$ ) such that $\frac{d \mathcal{L}}{d t} \leqslant-\delta n^{2 / 3} \mathcal{L}$, which gives the conclusion.

Proof of Proposition 15: Working as in the proof of Proposition 12, we get

$$
\mathcal{C}_{3} e^{-c^{*} \sqrt{n}} \int_{T / 3}^{2 T / 3} \int_{\beta}^{1}\left(\sqrt{n}\left|\partial_{v} g\right|^{2}+n^{3 / 2}|g|^{2}\right) d v d t \leqslant \mathcal{C}_{4} \int_{0}^{T} \int_{\beta}^{a}|g|^{2} d v d t
$$


for $n$ large enough. Moreover, thanks to Proposition 16, we have, for any $t \in$ $(T / 3,2 T / 3)$,

$$
\begin{aligned}
& \int_{\beta}^{1}\left(\sqrt{n}\left|\partial_{v} g(t, v)\right|^{2}+n^{3 / 2}|g(t, v)|^{2}\right) d v \\
\geqslant & n^{7 / 6} \int_{\beta}^{1}\left(\frac{1}{n^{2 / 3}}\left|\partial_{v} g(t, v)\right|^{2}+|g(t, v)|^{2}\right) d v \\
\geqslant & \frac{n^{7 / 6}}{K} e^{\delta n^{2 / 3} \frac{T}{3}} \int_{\beta}^{1}|g(T, v)|^{2} d v .
\end{aligned}
$$

Thus,

$$
\int_{\beta}^{1}|g(T)|^{2} d v \leqslant \frac{\mathcal{C}_{5}}{n^{7 / 6} T} e^{\left[c^{*} \sqrt{n}-\delta n^{3 / 2} T / 3\right]} \int_{0}^{T} \int_{\beta}^{a}|g|^{2} d v d t
$$

where $\mathcal{C}_{5}:=3 K \mathcal{C}_{4} / \mathcal{C}_{3}$, which gives the conclusion.

\subsubsection{Cut-off strategy}

Let $\xi_{i} \in C^{\infty}(\mathbb{R})$ for $i=1,2,3$ such that $0 \leqslant \xi_{i} \leqslant 1$ and

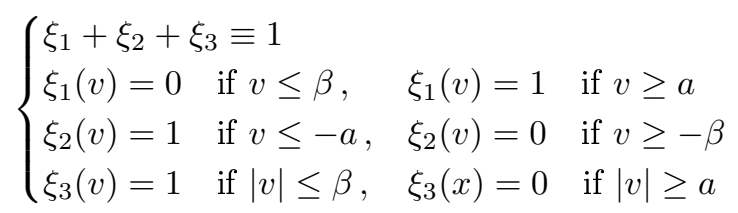

By Proposition 14, there exists $u_{1} \in L^{2}\left((0, T) \times \Omega_{1}\right)$ such that the solution of

$$
\begin{cases}\partial_{t} f_{1}+v^{2} \partial_{x} f_{1}-\partial_{v}^{2} f_{1}=u_{1}(t, x, v) 1_{\omega_{1}}(x, v), & (t, x, v) \in(0, T) \times \Omega_{1}, \\ f_{1}(t, x, \beta)=f_{1}(t, x, 1)=0, & (t, x) \in(0, T) \times \mathbb{T}, \\ f_{1}(0, x, v)=f_{0}(x, v), & (x, v) \in \Omega_{1},\end{cases}
$$

satisfies $f_{1}(T, .,)=$.0 . Similarly, let $\Omega_{2}:=\mathbb{T} \times(-1,-\beta), \omega_{2}:=\mathbb{T} \times(-a,-\beta)$; there exists $u_{2} \in L^{2}\left((0, T) \times \Omega_{2}\right)$ such that the solution of

$$
\begin{cases}\partial_{t} f_{2}+v^{2} \partial_{x} f_{2}-\partial_{v}^{2} f_{2}=u_{2}(t, x, v) 1_{\omega_{2}}(x, v), & (t, x, v) \in(0, T) \times \Omega_{2}, \\ f_{2}(t, x,-1)=f_{2}(t, x,-\beta)=0, & (t, x) \in(0, T) \times \mathbb{T}, \\ f_{2}(0, x, v)=f_{0}(x, v), & (x, v) \in \Omega_{2},\end{cases}
$$

satisfies $f_{2}(T, .,)=$.0 . Finally, let $\Omega_{3}:=\mathbb{T} \times(-\beta, \beta)$ and $f_{3}$ be the solution of

$$
\begin{cases}\partial_{t} f_{3}+v^{2} \partial_{x} f_{3}-\partial_{v}^{2} f_{3}=0, & (t, x, v) \in(0, T) \times \Omega_{3}, \\ f_{3}(t, x, \pm \beta)=0, & (t, x) \in(0, T) \times \mathbb{T}, \\ f_{3}(0, x, v)=f_{0}(x, v), & (x, v) \in \Omega_{3} .\end{cases}
$$

We extend $f_{j}$ and $u_{j}$ by zero on $(0, T) \times\left[\Omega-\Omega_{j}\right]$ for $j=1,2,3$. Then, the functions

$$
\begin{aligned}
f(t, x, v):=\xi_{1}(v) & f_{1}(t, x, v)+\xi_{2}(v) f_{2}(t, x, v)+\frac{T-t}{T} \xi_{3}(v) f_{3}(t, x, v), \\
u(t, x, v):= & \sum_{j=1}^{2} \xi_{j}(v) u_{j}(t, x, v) 1_{\omega_{j}}(x, v)-\frac{1}{T} \xi_{3}(v) f_{3}(t, x, v) \\
& -\sum_{j=1}^{3}\left(2 \xi_{j}^{\prime}(v) \partial_{v} f_{j}(t, x, v)+\xi_{j}^{\prime \prime}(v) f_{j}(t, x, v)\right)
\end{aligned}
$$

solve (1)-(3)-(4), as well as $f(T, . .)=$.0 on $\Omega$. 


\section{Proof of Theorem 1 with $\gamma=1$ and Dirichlet boundary conditions}

In all this section, we take $\gamma=1$ and $\omega=\mathbb{T} \times(a, b)$ where $-1<a<b<1$. Our goal is the proof of the statement 2 of Theorem 1. The strategy is the same as in the previous sections, it relies on the following dissipation property.

Proposition 17. There exists $K, \delta>0$ such that, for every $n \in \mathbb{Z}-\{0\}$ and $g_{0, n} \in H^{1}(-1,1)$, the solution of (15) satisfies

$$
\left\|g_{n}(t)\right\|_{L^{2}(-1,1)} \leqslant K e^{-\delta|n|^{2 / 3} t}\left\|g_{0, n}\right\|_{H^{1}(-1,1)}, \quad \forall t>0 .
$$

Moreover, the power " $2 / 3$ "in the exponential rate is optimal as $n \rightarrow+\infty$, and necessarily $\delta \leqslant \frac{\mu}{2}$, where $\mu$ is the first zero (from the right) of Airy function in the half line $(-\infty, 0)$.

The proof of Proposition 17 relies on the following result.

Proposition 18. There exists $B, C, \delta>0$ with $B^{2}<C$ such that, for every $L>0$ and $h_{0} \in L^{2}(-L, L)$, the solution of

$$
\begin{cases}\partial_{\tau} h=\partial_{y}^{2} h+i y h, & (\tau, y) \in(0,+\infty) \times(-L, L), \\ h(\tau, \pm L)=0, & \tau \in(0,+\infty) \\ h(0, y)=h_{0}(y), & y \in(-L, L)\end{cases}
$$

satisfies

$$
\mathcal{L}(\tau) \leqslant \mathcal{L}(0) e^{-\delta \tau}, \forall \tau>0
$$

where

$$
\mathcal{L}(\tau):=\int_{-L}^{L}\left(|h(\tau, y)|^{2}-2 B \Im\left[\overline{h(\tau, y)} \partial_{y} h(\tau, y)\right]+C\left|\partial_{y} h(\tau, y)\right|^{2}\right) d y .
$$

Proof of Proposition 18: Let $B, C>0$ be such that

$$
4 B^{2}<C \quad \text { and } \quad 2 C^{2}<B
$$

(for instance, $B=\sqrt{C} / 3$ with $C>0$ small enough). Easy computations show that

$$
\frac{1}{2} \frac{d \mathcal{L}}{d \tau}=-B\|h\|^{2}-C \Im\left[\int_{-L}^{L} h \partial_{y} \bar{h}\right]-\left\|\partial_{y} h\right\|^{2}-2 B \Im\left[\int_{-L}^{L} \partial_{y}^{2} \bar{h} \partial_{y} h\right]-C\left\|\partial_{y}^{2} h\right\|^{2} .
$$

Thanks to

$$
\begin{gathered}
-2 B \Im\left[\int_{-L}^{L} \partial_{y}^{2} \bar{h} \partial_{y} h\right] \leqslant C\left\|\partial_{y}^{2} h\right\|^{2}+\frac{B^{2}}{C}\left\|\partial_{y} h\right\|^{2}, \\
-C \Im\left[\int_{-L}^{L} h \partial_{y} \bar{h}\right] \leqslant \frac{B}{2}\|h\|^{2}+\frac{C^{2}}{2 B}\left\|\partial_{y} h\right\|^{2}
\end{gathered}
$$

we get

$$
\frac{d \mathcal{L}}{d \tau} \leqslant-\frac{B}{2}\|h\|^{2}-\left(1-\frac{B^{2}}{C}-\frac{C^{2}}{2 B}\right)\left\|\partial_{y} h\right\|^{2}
$$


Thanks to (32), there exists $\delta>0$ such that $d \mathcal{L} / d t \leqslant-\delta \mathcal{L}$, which gives the conclusion. $\square$

\section{Proof of Proposition 17:}

First step: Proof of (29): One may assume that $n>0$, otherwise, consider $\overline{g_{n}}$. In order to simplify the notation, we write $g$, instead of $g_{n}$. The function $h(\tau, y)$ defined by

$$
g(t, v)=h\left(n^{\frac{2}{3}} t, n^{\frac{1}{3}} v\right)
$$

satisfies (30) with $L=n^{\frac{1}{3}}$ and $h_{0}(y):=g_{0, n}\left(y / n^{\frac{1}{3}}\right)$. From the previous proposition, we know that

$$
\widetilde{\mathcal{L}}(t):=\int_{-1}^{1}\left(|g(t, v)|^{2}-\frac{2 B}{n^{\frac{1}{3}}} \Im\left[\overline{g(t, v)} \partial_{v} g(t, v)\right]+\frac{C}{n^{\frac{2}{3}}}\left|\partial_{v} g(t, v)\right|^{2}\right) d v
$$

satisfies $\widetilde{\mathcal{L}}(t) \leqslant \widetilde{\mathcal{L}}(0) e^{-\delta n^{\frac{2}{3}} t}$. Moreover, using $B^{2}<C$ we get

$$
\begin{aligned}
\int_{-1}^{1}\left|g_{n}(t, v)\right|^{2} d v & \leqslant\left(1-\frac{B^{2}}{C}\right)^{-1} \widetilde{\mathcal{L}}(t) \\
& \leqslant\left(1-\frac{B^{2}}{C}\right)^{-1} \widetilde{\mathcal{L}}(0) e^{-\delta n^{\frac{2}{3}} t} \\
& \leqslant K e^{-\delta n^{2 / 3} t}\left\|g_{0, n}\right\|_{H^{1}}^{2} .
\end{aligned}
$$

for some constant $K>0$.

Second step: Proof of the optimality. First, let us recall that the function

$$
\varphi(y):=\operatorname{Ai}\left(e^{i \frac{\pi}{6}} y+\mu\right)
$$

satisfies

$$
\begin{aligned}
& \left\{\begin{array}{l}
-\varphi^{\prime \prime}(y)+i y \varphi(y)=\lambda \varphi(y), \quad y \in(0,+\infty) \\
\varphi(0)=0,
\end{array}\right. \\
& |\varphi(y)| \leqslant \frac{C}{y^{1 / 4}} e^{-\frac{\sqrt{2}}{3} y^{3 / 2}}, \quad \forall y \in(0,+\infty),
\end{aligned}
$$

where $\lambda:=-e^{i \frac{2 \pi}{3}} \mu, C>0$ (see $[3$, formulas (2.12) and (A.12)]).

Working by contradiction, we assume that there exists $T>0, n^{*} \in \mathbb{N}^{*}$, $\left(r_{n}\right)_{n \in \mathbb{Z}} \in(0,+\infty)^{\mathbb{Z}}$ such that

- any solution of (15) satisfies

$$
\left\|g_{n}(t)\right\|_{L^{2}(-1,1)} \leqslant K e^{-r_{n} t}\left\|g_{0, n}\right\|_{H^{1}(-1,1)}, \quad \forall t \in[0, T], n \in \mathbb{Z},
$$

- $r_{n}>\left(\lambda_{r}+\delta\right)|n|^{2 / 3}, \forall|n| \geqslant n^{*}$, where $\lambda_{r}:=\Re(\lambda)=|\mu| / 2$ and $\delta>0$.

Let us consider $n \in \mathbb{N}^{*}$ and $\theta \in C_{c}^{\infty}(\mathbb{R})$ such that $\theta( \pm 1)=1$. Thanks to (33), the function

$$
\tilde{g}_{n}(t, v):=\left(\varphi\left(n^{\frac{1}{3}}(v+1)\right)-\varphi\left(2 n^{\frac{1}{3}}\right) \theta(v)\right) e^{-\left(\lambda n^{2 / 3}-i n\right) t}
$$

satisfies

$$
\begin{cases}\partial_{t} \tilde{g}_{n}+i n v \tilde{g}_{n}-\partial_{v}^{2} \tilde{g}_{n}=F_{n}, & (t, v) \in(0,+\infty) \times(-1,1), \\ \tilde{g}_{n}(t, \pm 1)=0, & t \in(0,+\infty),\end{cases}
$$


where

$$
F_{n}(t, v):=\varphi\left(2 n^{\frac{1}{3}}\right)\left(\left[\lambda n^{2 / 3}-i n(v+1)\right] \theta(v)+\theta^{\prime \prime}(v)\right) e^{-\left(\lambda n^{2 / 3}-i n\right) t} .
$$

Thanks to (34), there exists $C_{1}>0$ such that, for every $n \in \mathbb{N}^{*}$ and $t \in \mathbb{R}$,

$$
\left\|F_{n}(t)\right\|_{H^{1}} \leqslant C_{1} n^{\frac{11}{12}} e^{-\frac{4 \sqrt{n}}{3}-\lambda_{r} n^{\frac{2}{3}} t} .
$$

Thanks to the Duhamel formula and (34) we get the following inequality, for every $n \geqslant n^{*}$ and $t \in[0, T]$

$$
\left\|\tilde{g}_{n}(t)\right\|_{L^{2}} \leqslant K e^{-r_{n} t}\left\|\tilde{g}_{n}(0)\right\|_{H^{1}}+C_{2} n^{\frac{11}{12}} e^{-\frac{4 \sqrt{n}}{3}} \int_{0}^{t} e^{-r_{n}(t-s)-\lambda_{r} n^{\frac{2}{3}} s} d s,
$$

where $C_{2}>0$. Thus, there exists a constant $C_{3}>0$ such that, for every $n \geqslant n^{*}$ and $t \in[0, T]$

$$
e^{-\lambda_{r} n^{\frac{2}{3}} t} \leqslant C_{3}\left(e^{-r_{n} t}+R(n) e^{-\frac{4 \sqrt{n}}{3}} e^{-\lambda_{r} n^{\frac{2}{3}} t}\right),
$$

where $R$ is a rational fraction. We get a contradiction by considering the limit $n \rightarrow+\infty$ (with a fixed $t \in(0, T]$ ). This ends the proof of the optimality.

Remark 2. The optimality of $n^{2 / 3}$ in the exponential rate shows that, we cannot expect to prove the null controllability of (1)-(3) in the same way as we did for (1)-(2). Indeed, the dissipation in $e^{-\delta n^{2 / 3} t}$ is not sufficient to compensate for the constant $e^{C n}$ of Proposition 5. Therefore, with Dirichlet boundary conditions, if the null controllability holds with arbitrarily small control supports $\omega$, the proof requires another strategy.

\section{Conclusion and open problems}

In this article, we have studied the null controllability of Kolmogorov type equations (1), with $\gamma \in\{1,2\}$, in the rectangle $\Omega=\mathbb{T} \times(-1,1)$, with a distributed control localized on an open subset $\omega$ of $\Omega$.

The following questions are still open.

1. When $\gamma>2$, does null controllability hold? In [4], the proof of the non uniform observability relies on a comparison argument (maximum principle), which cannot be used here because the 1D heat equation has complex valued coefficients.

2. When $\gamma=2$, what is the value of the minimal time $T^{*}$ ? We conjecture that $T^{*}=a^{2} / 2$.

3. What happens for $\gamma \in(1,2)$ ? (with $v$ replaced by $|v|$ )

4. With $\gamma=1$ and Dirichlet boundary conditions in $v$, does null controllability hold with an arbitrary control support $\omega$ ? 
5. Is it possible to extend these results to multidimensional configurations? The technique of this paper should possibly extend to cylindrical domains of the form $\mathbb{T} \times(-1,1)^{m}$. However, the generalization to more general configurations or boundary controls, is widely open.

\section{Acknowledgments}

The author warmly thanks Philippe Gravejat, François Golse and Thierry Paul for stimulating discussions.

\section{A Proof of Proposition 1}

Let $a^{\prime}, b^{\prime}$ be such that $-1<a<a^{\prime}<b^{\prime}<b<1$. All the computations of the proof will be made assuming, first, $g \in H^{1}\left(0, T ; L^{2}(-1,1)\right) \cap L^{2}\left(0, T ; H^{2} \cap\right.$ $\left.H_{0}^{1}(-1,1)\right)$. Then, the conclusion of Proposition 1 will follow by a density argument.

Consider the weight function

$$
\alpha(t, v):=\frac{M \beta(v)}{t(T-t)}, \quad(t, v) \in(0, T) \times \mathbb{R},
$$

where $\beta \in C^{2}([-1,1])$ satisfies

$$
\begin{gathered}
\beta \geqslant 1 \text { on }(-1,1), \\
\left|\beta^{\prime}\right|>0 \text { on }\left[-1, a^{\prime}\right] \cup\left[b^{\prime}, 1\right], \\
\beta^{\prime}(1)>0, \quad \beta^{\prime}(-1)<0, \\
\beta^{\prime \prime}<0 \text { on }\left[-1, a^{\prime}\right] \cup\left[b^{\prime}, 1\right]
\end{gathered}
$$

and $M=M(T, n, \beta)>0$ will be chosen later on. We also introduce the function

$$
z(t, v):=g(t, v) e^{-\alpha(t, v)},
$$

that satisfies

$$
e^{-\alpha} \mathcal{P}_{n} g=P_{1} z+P_{2} z+P_{3} z
$$

where

$$
\begin{gathered}
P_{1} z:=-\frac{\partial^{2} z}{\partial v^{2}}+\left(\alpha_{t}-\alpha_{v}^{2}\right) z, \quad P_{2} z:=\frac{\partial z}{\partial t}-2 \alpha_{v} \frac{\partial z}{\partial v}+i n v^{\gamma} z \\
P_{3} z:=-\alpha_{v v} z .
\end{gathered}
$$

We develop the classical proof (see [25]), taking the $L^{2}(Q)$-norm in the identity (41), then developing the double product, which leads to

$$
\int_{Q}\left(\Re\left[P_{1} z \overline{P_{2} z}\right]-\frac{1}{2}\left|P_{3} z\right|^{2}\right) d v d t \leqslant \int_{Q}\left|e^{-\alpha} \mathcal{P}_{n} g\right|^{2} d v d t,
$$

where $Q:=(0, T) \times(-1,1)$. After computations (see [5] for details, we get

$$
\begin{aligned}
& \int_{Q}|z|^{2}\left\{-\frac{1}{2}\left(\alpha_{t}-\alpha_{v}^{2}\right)_{t}+\left[\left(\alpha_{t}-\alpha_{v}^{2}\right) \alpha_{v}\right]_{v}-\frac{1}{2} \alpha_{v v}^{2}\right\} d v d t \\
& +\int_{Q}\left\{n \gamma v^{\gamma-1} \Im\left(\bar{z} \frac{\partial z}{\partial v}\right)-\alpha_{v v}\left|\frac{\partial z}{\partial v}\right|^{2} \alpha_{v v}\right\} d v d t \leqslant \int_{Q}\left|e^{-\alpha} \mathcal{P}_{n} g\right|^{2} d v d t
\end{aligned}
$$


Now, in the left hand side of $(44)$ we separate the terms on $(0, T) \times\left(a^{\prime}, b^{\prime}\right)$ and those on $(0, T) \times\left[\left(-1, a^{\prime}\right) \cup\left(b^{\prime}, 1\right)\right]$. One has

$$
\begin{gathered}
-\alpha_{v v}(t, v) \geqslant \frac{C_{1} M}{t(T-t)} \quad \forall v \in\left[-1, a^{\prime}\right] \cup\left[b^{\prime}, 1\right], t \in(0, T), \\
\left|\alpha_{v v}(t, v)\right| \leqslant \frac{C_{2} M}{t(T-t)} \quad \forall v \in\left[a^{\prime}, b^{\prime}\right], t \in(0, T),
\end{gathered}
$$

where $C_{1}=C_{1}(\beta):=\min \left\{-\beta^{\prime \prime}(x) ; x \in\left[-1, a^{\prime}\right] \cup\left[b^{\prime}, 1\right]\right\}$ is positive thanks to the assumption (39) and $C_{2}=C_{2}(\beta):=\sup \left\{\left|\beta^{\prime \prime}(x)\right| ; x \in\left[a^{\prime}, b^{\prime}\right]\right\}$. Moreover,

$$
\begin{aligned}
& -\frac{1}{2}\left(\alpha_{t}-\alpha_{v}^{2}\right)_{t}+\left[\left(\alpha_{t}-\alpha_{v}^{2}\right) \alpha_{v}\right]_{v}-\frac{1}{2} \alpha_{v v}^{2}=\frac{1}{(t(T-t))^{3}}\left\{M \beta\left(3 T t-T^{2}-3 t^{2}\right)\right. \\
& \left.+M^{2}\left[(2 t-T)\left(\beta^{\prime \prime} \beta+2 \beta^{\prime 2}\right)-\frac{t(T-t) \beta^{\prime \prime 2}}{2}\right]-3 M^{3} \beta^{\prime \prime} \beta^{\prime 2}\right\} .
\end{aligned}
$$

Hence, owing to (37) and (39), there exist $m_{1}=m_{1}(\beta)>0 C_{3}=C_{3}(\beta)>0$ and $C_{4}=C_{4}(\beta)>0$ such that, for every $M \geqslant M_{1}$ and $t \in(0, T)$,

$$
\begin{gathered}
-\frac{1}{2}\left(\alpha_{t}-\alpha_{v}^{2}\right)_{t}+\left[\left(\alpha_{t}-\alpha_{v}^{2}\right) \alpha_{v}\right]_{v}-\frac{1}{2} \alpha_{v v}^{2} \geqslant \frac{C_{3} M^{3}}{[t(T-t)]^{3}} \quad \forall v \in\left[0, a^{\prime}\right] \cup\left[b^{\prime}, 1\right], \\
\left|-\frac{1}{2}\left(\alpha_{t}-\alpha_{v}^{2}\right)_{t}+\left[\left(\alpha_{t}-\alpha_{v}^{2}\right) \alpha_{v}\right]_{v}-\frac{1}{2} \alpha_{v v}^{2}\right| \leqslant \frac{C_{4} M^{3}}{[t(T-t)]^{3}} \quad \forall v \in\left[a^{\prime}, b^{\prime}\right]
\end{gathered}
$$

where

$$
M_{1}=M_{1}(T, \beta):=m_{1}(\beta)\left(T+T^{2}\right) .
$$

Using (44), (45) and (46), we deduce, for every $M \geqslant M_{1}$,

$$
\begin{aligned}
& \int_{0}^{T} \int_{\left(-1, a^{\prime}\right) \cup\left(b^{\prime}, 1\right)} \frac{C_{1} M}{t(T-t)}\left|\frac{\partial z}{\partial v}\right|^{2} d v d t \\
& \quad+\int_{0}^{T} \int_{\left(-1, a^{\prime}\right) \cup\left(b^{\prime}, 1\right)}\left[\frac{C_{3} M^{3}}{(t(T-t))^{3}}|z|^{2}-|n| \gamma|v|^{\gamma-1} \Im\left(\frac{\partial z}{\partial v} \bar{z}\right)\right] d v d t \\
& \leqslant \int_{0}^{T} \int_{a^{\prime}}^{b^{\prime}}\left[\frac{C_{2} M}{t(T-t)}\left|\frac{\partial z}{\partial x}\right|^{2}+\frac{C_{4} M^{3}}{(t(T-t))^{3}}|z|^{2}+|n| \gamma v^{\gamma-1} \Im\left(\frac{\partial z}{\partial v} \bar{z}\right)\right] d v d t \\
& +\int_{Q}\left|e^{-\alpha} \mathcal{P}_{n} g\right|^{2} d v d t .
\end{aligned}
$$

Let

$$
M_{2}=M_{2}(T, \beta):=\frac{T^{2} \sqrt{|n| \gamma}}{4 \sqrt[4]{C_{1} C_{3}}} .
$$

When $M \geqslant M_{2}$, we have

$$
\begin{aligned}
\left|n \gamma v^{\gamma-1} \Im\left(\frac{\partial z}{\partial v} \bar{z}\right)\right| & \leqslant \frac{1}{2} \frac{C_{3} M^{3}}{(t(T-t))^{3}}|z|^{2}+\frac{1}{2} \frac{(t(T-t))^{3}}{C_{3} M^{3}} \gamma^{2} n^{2}\left|\frac{\partial z}{\partial v}\right|^{2} \\
& \leqslant \frac{1}{2} \frac{C_{3} M^{3}}{(t(T-t))^{3}}|z|^{2}+\frac{C_{1} M}{2 t(T-t)}\left|\frac{\partial z}{\partial v}\right|^{2},
\end{aligned}
$$

because

$$
\begin{aligned}
\frac{1}{2} \frac{(t(T-t))^{3}}{C_{3} M^{3}} \gamma^{2} n^{2} & =\frac{C_{1} M}{2 t(T-t)} \frac{(t(T-t))^{4} \gamma^{2} n^{2}}{C_{1} C_{3} M^{4}} \\
& \leqslant \frac{C_{1} M}{2 t(T-t)} \frac{\left(T^{2} / 4\right)^{3} \gamma^{2} n^{2}}{C_{1} C_{3} M^{4}} \\
& =\frac{C_{1} M}{2 t(T-t)} \frac{M_{2}^{2}}{M^{4}}
\end{aligned}
$$


From now on, we take

$$
M=M(T, n, \beta):=\mathcal{C}_{2} \max \left\{T+T^{2} ; \sqrt{|n|} T^{2}\right\}
$$

where

$$
\mathcal{C}_{2}=\mathcal{C}_{2}(\beta):=\max \left\{m_{1} ; \frac{1}{4 \sqrt[4]{C_{1} C_{3}}}\right\}
$$

so that $M \geqslant M_{1}$ and $M_{2}$ (see (47) and (49)). We have

$$
\begin{aligned}
& \int_{0}^{T} \int_{\left(0-1, a^{\prime}\right) \cup\left(b^{\prime}, 1\right)}\left(\frac{C_{1} M}{2 t(T-t)}\left|\frac{\partial z}{\partial v}\right|^{2}+\frac{C_{3} M^{3}}{2(t(T-t))^{3}}|z|^{2}\right) d v d t \\
& \leqslant \int_{0}^{T} \int_{a^{\prime}}^{b^{\prime}}\left(\frac{C_{2}^{\prime} M}{t(T-t)}\left|\frac{\partial z}{\partial v}\right|^{2}+\frac{C_{6} M^{3}}{(t(T-t))^{3}}|z|^{2}\right) d v d t+\int_{Q}\left|e^{-\alpha} \mathcal{P}_{n} g\right|^{2} d v d t
\end{aligned}
$$

where $C_{6}=C_{6}(\beta):=C_{4}+C_{3} / 2, C_{2}^{\prime}=C_{2}(\beta)=C_{2}+C_{1} / 2$. Since for every $\epsilon>0$

$$
\begin{aligned}
& \frac{C_{1} M}{2 t(T-t)}\left|\frac{\partial g}{\partial x}-\alpha_{x} g\right|^{2}+\frac{C_{3} M^{3}}{2(t(T-t))^{3}}|g|^{2} \\
& \quad \geqslant\left(1-\frac{1}{1+\epsilon}\right) \frac{C_{1} M}{2 t(T-t)}\left|\frac{\partial g}{\partial x}\right|^{2}+\frac{M^{3}}{2(t(T-t))^{3}}\left(C_{3}-\epsilon C_{1}\left(\beta^{\prime}\right)^{2}\right)|g|^{2}
\end{aligned}
$$

and choosing

$$
\epsilon=\epsilon(\beta):=\frac{C_{3}}{2 C_{1}\left\|\beta^{\prime}\right\|_{\infty}^{2}},
$$

from (52), (53) and (40) we deduce that

$$
\begin{aligned}
& \int_{0}^{T} \int_{\left(0, a^{\prime}\right) \cup\left(b^{\prime}, 1\right)}\left(\frac{C_{7} M}{t(T-t)}\left|\frac{\partial g}{\partial v}\right|^{2}+\frac{C_{3} M^{3}|g|^{2}}{4(t(T-t))^{3}}\right) e^{-2 \alpha} d v d t \\
& \leqslant \int_{Q}\left|e^{-\alpha} \mathcal{P}_{n} g\right|^{2} d v d t+\int_{0}^{T} \int_{a^{\prime}}^{b^{\prime}}\left(\frac{C_{9} M^{3}|g|^{2}}{(t(T-t))^{3}}+\frac{C_{8} M}{t(T-t)}\left|\frac{\partial g}{\partial v}\right|^{2}\right) e^{-2 \alpha} d v d t
\end{aligned}
$$

where $C_{7}=C_{7}(\beta):=[1-1 /(1+\epsilon)] C_{1} / 2, C_{8}=C_{8}(\beta):=2 C_{2}^{\prime}$ and $C_{9}=C_{9}(\beta):=$ $C_{6}+2 C_{2}^{\prime} \sup \left\{\beta^{\prime}(x)^{2} ; x \in\left[a^{\prime}, b^{\prime}\right]\right\}$. Adding the same quantity in both sides, we get

$$
\begin{aligned}
\int_{Q}\left(\frac{C_{7} M}{t(T-t)}\left|\frac{\partial g}{\partial v}\right|^{2}\right. & \left.+\frac{C_{3} M^{3}|g|^{2}}{4(t(T-t))^{3}}\right) e^{-2 \alpha} d v d t \leqslant \int_{Q}\left|e^{-\alpha} \mathcal{P}_{n} g\right|^{2} d v d t \\
& +\int_{0}^{T} \int_{a^{\prime}}^{b^{\prime}}\left(\frac{C_{11} M^{3}|g|^{2}}{(t(T-t))^{3}}+\frac{C_{10} M}{t(T-t)}\left|\frac{\partial g}{\partial v}\right|^{2}\right) e^{-2 \alpha} d v d t
\end{aligned}
$$

where $C_{10}=C_{10}(\beta):=C_{8}+C_{7}$ and $C_{11}=C_{11}(\beta):=C_{9}+C_{3} / 4$. Thanks to a cut-off function $\rho$ such that

$$
0 \leqslant \rho \leqslant 1, \quad \rho \equiv 1 \text { on }\left(a^{\prime}, b^{\prime}\right), \quad \operatorname{Supp}(\rho) \subset(a, b)
$$


it is classic to get

$$
\begin{aligned}
\int_{0}^{T} \int_{a^{\prime}}^{b^{\prime}} \frac{C_{10} M}{t(T-t)}\left|\frac{\partial g}{\partial v}\right|^{2} & e^{-2 \alpha} d v d t \\
& \leqslant \int_{Q}\left|\mathcal{P}_{n} g\right|^{2} e^{-2 \alpha} d v d t+\int_{0}^{T} \int_{a}^{b} \frac{C_{12} M^{3}|g|^{2} e^{-2 \alpha}}{(t(T-t))^{3}} d v d t
\end{aligned}
$$

for some constant $C_{12}=C_{12}(\beta)>0$. Combining (55) with the previous inequality, we get

$$
\begin{aligned}
\int_{Q}\left(\frac{C_{7} M}{t(T-t)}\left|\frac{\partial g}{\partial v}\right|^{2}\right. & \left.+\frac{C_{3} M^{3}|g|^{2}}{4(t(T-t))^{3}}\right) e^{-2 \alpha} d v d t \\
& \leqslant \int_{Q} 2\left|e^{-\alpha} \mathcal{P}_{n} g\right|^{2} d v d t+\int_{0}^{T} \int_{a}^{b} \frac{C_{13} M^{3}|g|^{2}}{(t(T-t))^{3}} e^{-2 \alpha} d v d t
\end{aligned}
$$

where $C_{13}=C_{13}(\beta, \rho):=C_{11}+C_{12}$. Then, the global Carleman estimates (10) holds with

$$
\mathcal{C}_{1}=\mathcal{C}_{1}(\beta):=\frac{\min \left\{C_{7} ; C_{3} / 4\right\}}{\max \left\{2 ; C_{13}\right\}} .
$$

\section{References}

[1] F. Alabau-Boussouira, P. Cannarsa, and G. Fragnelli. Carleman estimates for degenerate parabolic operators with applications to null controllability. J. Evol. Equ., 6(2):161-204, 2006.

[2] S. Alinhac and C. Zuily. Uniqueness and nonuniqueness of the cauchy problem for hyperbolic operators with double characteristics. Comm. Partial Differential Equations, 6(7):799-828, 1981.

[3] Y. Almog. The stability of the normal state of superconductors in the presence of electric currents. SIAM J. Math. Anal., 40(2):824-850, 2008.

[4] K. Beauchard, P. Cannarsa, and R. Guglielmi. Null controllability of Grushin-type operators in dimension two. J. Eur. Math. Soc. (to appear), 2012.

[5] K. Beauchard and E. Zuazua. Some controllability results for the 2D Kolmogorov equation. Ann. Inst. H. Poincaré Anal. Non Linéaire, 26:1793$1815,2009$.

[6] A. Benabdallah, Y. Dermenjian, and J. Le Rousseau. Carleman estimates for the one-dimensional heat equation with a discontinuous coefficient and applications to controllability and an inverse problem. J. Math. Anal. Appl., 336(2):865-887, 2007.

[7] A. Benabdallah, Y. Dermenjian, and J. Le Rousseau. On the controllability of linear parabolic equations with an arbitrary control location for stratified media. C. R. Math. Acad. Sci. Paris, 344(6):357-362, 2007.

[8] H. Brézis. Analyse fonctionnelle, theorie et applications. Masson, Paris, 1983. 
[9] P. Cannarsa and L. de Teresa. Controllability of 1-d coupled degenerate parabolic equations. Electron. J. Differ. Equ., Paper No. 73:21 p., 2009.

[10] P. Cannarsa, G. Fragnelli, and D. Rocchetti. Null controllability of degenerate parabolic operators with drift. Netw. Heterog. Media, 2(4):695-715 (electronic), 2007.

[11] P. Cannarsa, G. Fragnelli, and D. Rocchetti. Controllability results for a class of one-dimensional degenerate parabolic problems in nondivergence form. J. Evol. Equ., 8:583-616, 2008.

[12] P. Cannarsa, P. Martinez, and J. Vancostenoble. Persistent regional null controllability for a class of degenerate parabolic equations. Commun. Pure Appl. Anal., 3(4):607-635, 2004.

[13] P. Cannarsa, P. Martinez, and J. Vancostenoble. Null controllability of degenerate heat equations. Adv. Differential Equations, 10(2):153-190, 2005.

[14] P. Cannarsa, P. Martinez, and J. Vancostenoble. Carleman estimates for a class of degenerate parabolic operators. SIAM J. Control Optim., 47(1):119,2008 .

[15] P. Cannarsa, P. Martinez, and J. Vancostenoble. Carleman estimates and null controllability for boundary-degenerate parabolic operators. $C$. $R$. Math. Acad. Sci. Paris, 347(3-4):147-152, 2009.

[16] A. Doubova, E. Fernández-Cara, and E. Zuazua. On the controllability of parabolic systems with a nonlinear term involving the state and the gradient. SIAM J. Control Optim., 42 (3):798-819, 2002.

[17] A. Doubova, A. Osses, and J.-P. Puel. Exact controllability to trajectories for semilinear heat equations with discontinuous diffusion coefficients. ESAIM Control Optim. Calc. Var., 8:621-661, 2002.

[18] Sylvain Ervedoza. Control and stabilization properties for a singular heat equation with an inverse-square potential. Comm. Partial Differential Equations, 33(10-12):1996-2019, 2008.

[19] C. Fabre, J.P. Puel, and E. Zuazua. Approximate controllability of the semilinear heat equation. Proc. Roy. Soc. Edinburgh, 125A:31-61, 1995.

[20] H.O. Fattorini and D. Russel. Exact controllability theorems for linear parabolic equations in one space dimension. Arch. Rational Mech. Anal., 43:272-292, 1971.

[21] E. Fernández-Cara and E. Zuazua. Null and approximate controllability for weakly blowing up semilinear heat equations. Ann. Inst. H. Poincaré Anal. Non Linéaire, 17:583-616, 2000.

[22] E. Fernández-Cara and E. Zuazua. The cost of approximate controllability for heat equations: The linear case. Advances in Differential Equations, $5(4-6): 465-514,2000$. 
[23] E. Fernández-Cara and E. Zuazua. On the null controllability of the onedimensional heat equation with BV coefficients. Computational and Applied Mathematics, 12:167-190, 2002.

[24] C. Flores and L. de Teresa. Carleman estimates for degenerate parabolic equations with first order terms and applications. C. R. Math. Acad. Sci. Paris, 348(7-8):391-396, 2010.

[25] A.V. Fursikov and O.Y. Imanuvilov. Controllability of evolution equations. Lecture Notes Series, Seoul National University Research Institute of Mathematics Global Analysis Research Center, Seoul, 34, 1996.

[26] M. González-Burgos and L. de Teresa. Some results on controllability for linear and nonlinear heat equations in unbounded domains. Adv. Differential Equations, 12 (11):1201-1240, 2007.

[27] L. Hörmander. Hypoelliptic second order differential equations. Acta Math., 119:147-171, 1967.

[28] O.Y. Imanuvilov. Boundary controllability of parabolic equations. Uspekhi. Mat. Nauk, 48(3(291)):211-212, 1993.

[29] O.Y. Imanuvilov. Controllability of parabolic equations. Mat. Sb., 186(6):109-132, 1995.

[30] O.Y. Imanuvilov and M. Yamamoto. Carleman estimate for a parabolic equation in sobolev spaces of negative order and its applications. Control of Nonlinear Distributed Parameter Systems, G. Chen et al. eds., MarcelDekker, pages 113-137, 2000.

[31] G. Lebeau and L. Robbiano. Contrôle exact de l'équation de la chaleur. Comm. P.D.E., 20:335-356, 1995.

[32] G. Lebeau and J. Le Rousseau. On Carleman estimates for elliptic and parabolic operators. Applications to unique continuation and control of parabolic equations. ESAIM:COCV, 18:712-747, 2012.

[33] A. Lopez and E. Zuazua. Uniform null controllability for the one dimensional heat equation with rapidly oscillating periodic density. Annales IHP. Analyse non linéaire, 19 (5):543-580, 2002.

[34] P. Martinez and J. Vancostenoble. Carleman estimates for one-dimensional degenerate heat equations. J. Evol. Equ., 6(2):325-362, 2006.

[35] P. Martinez, J. Vancostonoble, and J.-P. Raymond. Regional null controllability of a linearized Crocco type equation. SIAM J. Control Optim., 42, no. 2:709-728, 2003.

[36] L. Miller. On the null-controllability of the heat equation in unbounded domains. Bulletin des Sciences Mathématiques, 129, 2:175-185, 2005.

[37] L. Miller. On exponential observability estimates for the heat semigroup with explicit rates. Rendiconti Lincei: Matematica e Applicazioni, 17, 4:351-366, 2006. 
[38] A. Pazy. Semigroups of linear operators and applications to partial differential equations. Applied Mathematical Sciences, vol. 44, Springer-Verlag, New York, 1983.

[39] J. Le Rousseau. Carleman estimates and controllability results for the onedimensional heat equation with bv coefficients. J. Differential Equations, 233(2):417-447, 2007.

[40] J. Vancostenoble and E. Zuazua. Null controllability for the heat equation with singular inverse-square potentials. J. Funct. Anal., 254(7):1864-1902, 2008.

[41] C. Villani. Hypocoercivity. Mem. Amer. Math. Soc., vol 202, N. 950, 2009.

[42] E. Zuazua. Approximate controllability of the semilinear heat equation: boundary control. International Conference in honour of Prof. R. Glowinski, Computational Sciences for the 21st Century, M.O. Bristeau et al. eds., John Wiley and Sons, pages 738-747, 1997.

[43] E. Zuazua. Finite dimensional null-controllability of the semilinear heat equation. J.Math. Pures et Appl., 76:237-264, 1997. 